\title{
On the Formulation and Implementation of the Love's Constraint for the Source Reconstruction Method
}

\author{
Mario Phaneuf, Student Member, IEEE, Puyan Mojabi, Member, IEEE
}

\begin{abstract}
The formulation and implementation of the Love's condition constraint for the source reconstruction method (SRM) in near-field antenna measurements is analyzed in the context of inverse problems. To this end, the SRM is first analyzed to identify the non-unique or non-radiating current sources which may be present. Next, the advantages and disadvantages of general regularization techniques, which may address the nonradiating currents, are presented which serve to motivate the use of the Love's condition constraint. The main methods of formulating the constraint are then presented, one of which is a novel technique developed for this paper. Following this, the formulation methods are analyzed in order to predict the similarities and differences of the methods in the context of addressing the non-radiating currents of the SRM. This analysis is reinforced by simulated antenna measurements. In particular, the novel formulation method is demonstrated to provide greater reconstruction accuracy (in the examples considered), at the cost of computational complexity.
\end{abstract}

Index Terms-Antenna measurements, inverse problems

\section{INTRODUCTION}

$\mathbf{T}$ HE near-field antenna measurement [1] is a multi-faceted tool for antenna design. When used for antenna (far-field) characterization [2], the near-field data are used in conjunction with forward propagation techniques to obtain key far-field parameters, e.g., the radiation pattern. When used for antenna diagnostics [3], the near-field data are used in conjunction with backward propagation techniques to obtain the field in the immediate vicinity of the antenna, which can highlight any issues (e.g., a faulty element in an antenna array [4]).

Among these techniques, the source reconstruction method (SRM) is a technique which is useful for both characterization and diagnostics [5], [6]. The SRM uses the near-field measured data to reconstruct equivalent electric and magnetic currents which characterize the antenna according to the surface equivalence principle. Furthermore, these currents are directly related to the tangential field under Love's condition [7]; thus, the SRM also provides diagnostic information. One of the key advantages of the SRM is the ability to use arbitrary reconstruction and measurement surfaces, which can be useful in situations where canonical surfaces (e.g., closed sphere) are not practical. The tradeoff or disadvantage of such a method is the increased

The authors would like to acknowledge the financial support of NSERC of Canada, Canada Research Chair Program, and the University of Manitoba Graduate Fellowship Program.

The authors are with the Department of Electrical and Computer Engineering at the University of Manitoba, Winnipeg, MB, Canada. (E-mails: phaneuf3@myumanitoba.ca and Puyan.Mojabi@umanitoba.ca.) computational complexity, since the SRM represents an inverse source problem which must be approached carefully. In brief, the reconstructed currents from the SRM are not unique, e.g., non-radiating sources [8] exist. One method of addressing this issue is to make the explicit enforcement of Love's condition [7], [9]-[11], which is often implemented as a weighted side constraint in the optimization process. The inclusion of this constraint is effective at preventing any currents which do not satisfy Love's condition. The implementation of the Love's constraint is not fixed; both the exact numerical implementation and the mathematical formulation may differ (e.g., [7] versus [10]). However, the consequences of differences in formulation and implementation have not been thoroughly investigated.

One of the main purposes of this paper is to carefully examine the consequences of differing mathematical formulations for the Love's condition constraint. To this end, the existing formulation methods will be formally re-introduced in a standardized framework. Using this framework, the formulation methods will be analyzed in the context of inverse problems in order to predict the similarities and differences of each method. As a consequence of this procedure, a novel formulation technique will be introduced in order to complete the framework. This novel technique can potentially offer increased accuracy, at the cost of computational complexity. It will be proposed that the potential accuracy of a given method is dependent on the constraint's ability to limit the undesired non-unique or non-radiating sources. It will be shown that in ideal cases, defined herein, each formulation method results in an equivalent constraint. In non-ideal cases, e.g., undersampled data, the methods begin to differ with respect to the degree of restriction which is imposed on the non-unique or non-radiating sources. The similarities and differences of each formulation method will be examined for two antennas: an infinitesimal dipole antenna with synthetic data, and a circular horn antenna with simulated data from commercial software.

The outline of the paper is as follows. Section II will present the SRM, whose notation and convention will be followed throughout the paper. It will also discuss the possible nonunique or non-radiating sources which exist. Section III will present the general strategies for treating the non-unique or non-radiating sources (otherwise known as regularization), which will motivate the use of the Love's constraint. Section IV will formally present the different formulation methods for the Love's constraint. Section V will analyze these methods and will propose several conclusions regarding the effective- 


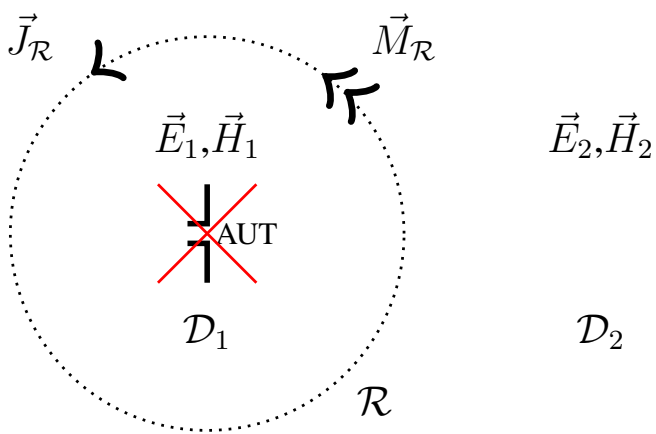

Fig. 1. Application of the equivalence principle for the equivalent problem in region $\mathcal{D}_{2}$. The equivalent surface currents on $\mathcal{R}$ generate the same fields in $\mathcal{D}_{2}$ as would the original AUT residing in $\mathcal{D}_{1}$.

ness of each method. Section VI will present the results for each method when applied to the infinitesimal dipole antenna and the circular horn antenna. Following this, Section VII will provide a discussion on the results to clarify and reinforce the main ideas of the paper. Finally, the conclusions of this paper will be summarized in Section VIII.

\section{Source Reconstruction Method (SRM)}

Consider two regions in free space: $\mathcal{D}_{1}$, which is enclosed by a closed surface $\mathcal{R}$ and contains the AUT, and $\mathcal{D}_{2}$, which comprises the space not occupied by the former. By the equivalence principle [12], an equivalent problem for $\mathcal{D}_{2}$ can be formulated by replacing the original AUT in $\mathcal{D}_{1}$ with arbitrary fields, as shown in Fig. 1. To support the fields in $\mathcal{D}_{2}$, and to maintain the electromagnetic boundary conditions [12], equivalent surface electric and magnetic currents $\vec{J}_{\mathcal{R}}$ and $\vec{M}_{\mathcal{R}}$ are introduced on the surface $\mathcal{R}$ which follow the relations

$$
\vec{J}_{\mathcal{R}}=\hat{n} \times\left(\vec{H}_{2}-\vec{H}_{1}\right), \quad \vec{M}_{\mathcal{R}}=-\hat{n} \times\left(\vec{E}_{2}-\vec{E}_{1}\right),
$$

where $\hat{n}$ is the outer normal unit vector on $\mathcal{R}$ (pointing into $\mathcal{D}_{2}$ ), $\vec{E}_{1}$ and $\vec{H}_{1}$ are the arbitrary electric and magnetic fields in $\mathcal{D}_{1}$, and finally $\vec{E}_{2}$ and $\vec{H}_{2}$ are the electric and magnetic fields in $\mathcal{D}_{2}$. (We have omitted the position vector $\vec{r}$ for simplicity.) The fields in $\mathcal{D}_{2}$ are now uniquely known by the knowledge of the equivalent currents on $\mathcal{R}$.

The choice of zero internal fields (i.e., $\vec{E}_{1}=\vec{H}_{1}=0$ ) corresponds to the well-known Love's equivalence principle or Love's condition [13]. In this case, the boundary conditions become

$$
\vec{J}_{\mathcal{R}}=\hat{n} \times \vec{H}_{2}, \quad \vec{M}_{\mathcal{R}}=-\hat{n} \times \vec{E}_{2} . \quad \text { (Love's Condition) }
$$

Under this relation, the equivalent currents are directly related to the tangential fields on $\mathcal{R}$, which can be useful in certain scenarios. Hereinafter, the currents in (2) may be referred to as the Love's currents.

Suppose that there is a measurement surface $\mathcal{S}$, upon which we obtain the measured fields $\vec{\Psi}_{\text {meas }}$. The equivalent currents are related to these fields as

$$
f_{G}\left(\vec{J}_{\mathcal{R}}, \vec{M}_{\mathcal{R}}\right)=\vec{\Psi}_{\text {meas }},
$$

where $f_{G}($.$) is the Green's function operator which operates$ on the currents to produce the external fields. The source reconstruction method [5], [6] intends to solve this equation for the equivalent currents. The Method of Moments (MoM) can be used to discretize (3) into a data equation

$$
\mathbf{A y}=\mathbf{b},
$$

where $\mathbf{b} \in \mathbb{C}^{M}$ is a vector containing the discrete measured data, A $\in \mathbb{C}^{M \times N}$ is a matrix representing the discrete Green's function operator, and $\mathbf{y} \in \mathbb{C}^{N}$ is a vector containing the appropriate basis function coefficients for the equivalent currents.

Since the SRM, and consequently (4), represent an inverse (source) problem, the solution is potentially subject to the following characteristics of inverse or ill-posed problems [14]:

1) the solution does not exist (non-existence),

2) the solution is not unique (non-uniqueness),

3) the solution is not stable (instability).

Note that since we are dealing with physical problems, the first item (non-existence) does not apply. The second item is represented by the concept of non-radiating (NR) currents in inverse source problems [8], or equivalently a non-trivial kernel or null-space for the operator A [15]. In other words, there exist currents $\mathbf{y}_{\mathrm{NR} 1}$ which satisfy

$$
\left\|\mathbf{A y}_{\mathrm{NR} 1}\right\|_{2}=0
$$

where $\|\cdot\|_{2}$ denotes the $L_{2}$ norm over the measurement domain $\mathcal{S}$. The third item arises in ill-conditioned problems where the presence of measurement and approximation error results in an overfitting of the matrix equation [14], [16]. In other words, there can be large erroneous currents included in the obtained solution as we attempt to match the inexact data. These currents $\mathbf{y}_{\mathrm{NR} 2}$ satisfy

$$
\left\|\mathbf{A} \mathbf{y}_{\mathrm{NR} 2}\right\|_{2} \leq \delta
$$

where $\delta \in \mathbb{R}^{+}$is a small scalar value. Since the presence of noise and error can mask the effect of these currents in the measured data $\mathbf{b}$, they have been denoted as nonradiating since this is effectively the case. In physical terms, these currents can represent currents which produce evanescent fields [12] (e.g., in the near-field zone of the antenna [17]). Alternatively, these currents represent the approximate nullspace [18] of the data operator. Hereinafter, we collectively refer to $\mathbf{y}_{\mathrm{NR} 1}$ and $\mathbf{y}_{\mathrm{NR} 2}$ as $\mathbf{y}_{\mathrm{NR}}$.

In general, a solution obtained by the SRM will take the form

$$
\mathbf{y}=\mathbf{y}_{\mathrm{R}}+\mathbf{y}_{\mathrm{NR}}^{\mathrm{p}},
$$

where $\mathbf{y}_{\mathrm{R}}$ are radiating currents which generate the measured data, and $\mathbf{y}_{\mathrm{NR}}^{\mathrm{p}}$ are arbitrary non-radiating currents. Since the latter are not unique, they represent a particular choice from the total set, i.e., $\mathbf{y}_{\mathrm{NR}}^{\mathrm{p}} \in\left\{\mathbf{y}_{\mathrm{NR}}\right\}$. The presence of an arbitrary $\mathbf{y}_{\mathrm{NR}}^{\mathrm{p}}$ in the solution may or may not be a problem for the overall antenna measurement. In the case of antenna diagnostics, we wish to relate the currents to the fields by (2); thus, the presence of non-radiating currents must be addressed. In the case of antenna characterization, the presence of an arbitrary $\mathbf{y}_{\mathrm{NR}}^{\mathrm{p}}$ is typically not a problem. If the measured data or fields on $\mathcal{S}$ are well-sampled over a closed surface, then by the electromagnetic uniqueness theorem, these fields, or the 
currents which produce them, will dictate a unique far-field. Thus, the non-radiating currents are not an issue. However, if the data are under-sampled, or the measurement surface is not closed, the set of non-radiating currents will be expanded to include currents which are not radiating with respect to $\mathcal{S}$ but are otherwise radiating. This can be understood again by the uniqueness theorem; since the field is not specified over a closed surface, the far-field cannot be uniquely dictated.

Since the Love's currents in (2) produce the unique field in $\mathcal{D}_{2}$, they will also produce the unique far-field. Thus, a solution which satisfies the purposes of antenna diagnostics and characterization is the Love's currents constructed with the true fields on $\mathcal{R}$. Herinafter, we will assume that the desired currents of the SRM are these currents.

\section{REGULARIZATION OF THE SRM}

When the presence of non-radiating currents needs to be controlled, we can employ the family of techniques commonly referred to as regularization. Many common techniques involve the addition of a weighted side constraint during the optimization process. If the constraint is represented by a matrix $\mathbf{A}_{2}$, then it is customary to solve the least-squares problem which is equivalent to minimizing [16]

$$
\min _{\mathbf{y}}\{\underbrace{\|\mathbf{A y}-\mathbf{b}\|_{2}^{2}}_{\text {data misfit }}+\alpha^{2} \underbrace{\left\|\mathbf{A}_{2} \mathbf{y}\right\|_{2}^{2}}_{\text {regularization }}\}
$$

where $\alpha$ is a weighting factor. This cost functional is known as Tikhonov's method [14]. The matrix $\mathbf{A}_{2}$ is often chosen as an identity matrix, which has the effect of favoring solutions with small norms. The identity Tikhonov strategy is equivalent to the (early) truncation of a conjugate gradient (CG) iterative method applied to (4) [19], [20], otherwise known as Krylov subspace regularization. Since the non-radiating currents are orthogonal to the radiating currents [21], $\left\|\mathbf{y}_{\mathrm{R}}+\mathbf{y}_{\mathrm{NR}}^{\mathrm{p}}\right\|_{2}^{2}=$ $\left\|\mathbf{y}_{\mathrm{R}}\right\|_{2}^{2}+\left\|\mathbf{y}_{\mathrm{NR}}^{\mathrm{p}}\right\|_{2}^{2} \geq\left\|\mathbf{y}_{\mathrm{R}}\right\|_{2}^{2}$; in other words, $\mathbf{y}_{\mathrm{R}}$ represents the minimum norm solution. Thus, the identity Tikhonov strategy will favor the removal of $\mathbf{y}_{\mathrm{NR}}$ currents; the degree of this removal is dictated by the regularization parameter $\alpha$.

The underlying issue to this discussion is the fact that the desired solution will likely contain non-radiating sources. Thus, we are not guaranteed to obtain the desired solution, regardless of the choice of $\alpha$ [14]. A potential resolution to this problem is to make a more informed choice of $\mathbf{A}_{2}$, e.g., we could incorporate auxiliary physical information whose constraint is satisfied by the desired solution. In Section II, the argument was made that the Love's currents in (2) are the desired solution for antenna diagnostics and also useful for antenna characterization. Thus, a logical choice of $\mathbf{A}_{2}$ would be that which enforces Love's condition. Under this choice, the desired Love's currents, which includes the desired nonradiating currents, will satisfy both the data equation and the constraint. Furthermore, a subset of $\left\{\mathbf{y}_{\mathrm{NR}}\right\}$, corresponding to undesired non-radiating currents, will likely be prevented by the Love's constraint. Note that we do not necessarily remove the complete set of undesired non-radiating currents, as there is nothing preventing a particular $\mathbf{y}_{\mathrm{NR}}^{\mathrm{p}}$ from independently satisfying the constraint. This can be rectified by further incorporating an identity Tikhonov constraint. Unlike the previous case, the set of non-radiating currents have already been restricted by the Love's condition and thus it will likely be much easier to choose the regularization weight. Herein, we will propose that the formulation and implementation of the Love's condition can affect the degree of this restriction, and that a larger restriction can better facilitate the procurement of the desired Love's currents.

The enforcement of Love's condition for antenna measurements was first proposed in [7]. Therein, two equivalent techniques are proposed to enforce Love's condition, of which only one is applied and evaluated. The first technique is the explicit enforcement of the classical jump conditions under Love's condition, which results in a relation between the equivalent currents which generate the field, and the very same currents which are related to the field under Love's condition. (This technique has since been demonstrated; for example, see [10], [11].) The second technique only considers the internal side of the jump condition, which can avoid the singular evaluation of the Green's function [7]. ${ }^{1}$ This can be implemented by introducing virtual measurement points on a surface just within $\mathcal{R}$ with a measured field of zero. Herein, we will show that the two methods are indeed equivalent in the ideal case but can differ in the practical case. In addition, we will propose a third method for the enforcement of Love's condition, which is equivalent to the other methods in the ideal case, but potentially more accurate in the practical case at the cost of computational complexity.

\section{ENFORCING LOVE's CONDITION}

In this section, the three methods of enforcing Love's condition will be presented and described.

\section{A. Jump Condition Method (JCM)}

The most fundamental of the three methods is the enforcement of the classical jump conditions on $\mathcal{R}$ under Love's condition [7], [10]. We will refer to this technique as the jump condition method (JCM). We begin by defining the operators $\mathcal{L}(\vec{u} ; \vec{r})$ and $\mathcal{K}(\vec{u} ; \vec{r})$ as [7]

$$
\mathcal{L}(\vec{u} ; \vec{r})=j k_{0} \int_{\mathcal{R}}\left[\vec{u}\left(\vec{r}^{\prime}\right)+\frac{1}{k_{0}^{2}} \nabla \nabla_{s}^{\prime} \cdot \vec{u}\left(\vec{r}^{\prime}\right)\right] g\left(\vec{r}, \vec{r}^{\prime}\right) d s^{\prime}
$$

and

$$
\mathcal{K}(\vec{u} ; \vec{r})=\int_{\mathcal{R}} \vec{u}\left(\vec{r}^{\prime}\right) \times \nabla g\left(\vec{r}, \vec{r}^{\prime}\right) d s^{\prime},
$$

where

$$
g\left(\vec{r}, \vec{r}^{\prime}\right)=\frac{e^{-j k_{0}\left|\vec{r}-\vec{r}^{\prime}\right|}}{4 \pi\left|\vec{r}-\vec{r}^{\prime}\right|} .
$$

The variable $\vec{r}$ is the position vector, $k_{0}$ is the wavenumber in free space, $\nabla f$ is the gradient operator on the scalar $f, \nabla_{s}^{\prime} \cdot \vec{f}$ is the surface divergence operator on the vector $\vec{f}, d s^{\prime}$ is a surface differential element on $\mathcal{R}$, and $g\left(\vec{r}, \vec{r}^{\prime}\right)$ is the scalar Green's function in free space. The external magnetic field

\footnotetext{
${ }^{1}$ In addition to the area of antenna measurements, this approach to enforce Love's condition has been used in other applications such as metasurface design [22] and specific absorption rate characterization [23].
} 
$\vec{H}(\vec{r})$ can be obtained from the equivalent currents $\vec{J}_{\mathcal{R}}$ and $\vec{M}_{\mathcal{R}}$ by [7]

$$
\vec{H}(\vec{r})=-\frac{1}{\eta_{0}} \mathcal{L}\left(\vec{M}_{\mathcal{R}} ; \vec{r}\right)-\mathcal{K}\left(\vec{J}_{\mathcal{R}} ; \vec{r}\right),
$$

where $\eta_{0}$ is the wave impedance in free space. Likewise, the external electric field $\vec{E}$ can be obtained by [7]

$$
\vec{E}(\vec{r})=-\eta_{0} \mathcal{L}\left(\vec{J}_{\mathcal{R}} ; \vec{r}\right)+\mathcal{K}\left(\vec{M}_{\mathcal{R}} ; \vec{r}\right) .
$$

When the position vector coincides with $\mathcal{R}$, i.e., $\vec{r} \in \mathcal{R}$, the classical jump conditions state that the field is the average field over the boundary [24], that is

$$
\begin{array}{ll}
\frac{\vec{H}_{2}(\vec{r})+\vec{H}_{1}(\vec{r})}{2}=-\frac{1}{\eta_{0}} \mathcal{L}\left(\vec{M}_{\mathcal{R}} ; \vec{r}\right)-\mathcal{K}\left(\vec{J}_{\mathcal{R}} ; \vec{r}\right) & \forall \vec{r} \in \mathcal{R}, \\
\frac{\vec{E}_{2}(\vec{r})+\vec{E}_{1}(\vec{r})}{2}=-\eta_{0} \mathcal{L}\left(\vec{J}_{\mathcal{R}} ; \vec{r}\right)+\mathcal{K}\left(\vec{M}_{\mathcal{R}} ; \vec{r}\right) \quad \forall \vec{r} \in \mathcal{R},
\end{array}
$$

where we have employed the region notation in Section II. Under Love's condition, the inner boundary field is zero, so the average field is half of the outer fields. Since Love's condition also gives $\hat{n} \times \vec{H}_{2}=\vec{J}_{\mathcal{R}}$ and $-\hat{n} \times \vec{E}_{2}=\vec{M}_{\mathcal{R}}$, these can be substituted into (14) and (15) as

$$
\frac{1}{2} \vec{J}_{\mathcal{R}}(\vec{r})=\hat{n} \times\left[-\frac{1}{\eta_{0}} \mathcal{L}\left(\vec{M}_{\mathcal{R}} ; \vec{r}\right)-\mathcal{K}\left(\vec{J}_{\mathcal{R}} ; \vec{r}\right)\right] \quad \forall \vec{r} \in \mathcal{R},
$$$$
\frac{1}{2} \vec{M}_{\mathcal{R}}(\vec{r})=-\hat{n} \times\left[-\eta_{0} \mathcal{L}\left(\vec{J}_{\mathcal{R}} ; \vec{r}\right)+\mathcal{K}\left(\vec{M}_{\mathcal{R}} ; \vec{r}\right)\right] \quad \forall \vec{r} \in \mathcal{R} .
$$

It is relatively straightforward to discretize the equations above under the MoM in order to form a set of discrete equations. If $\mathbf{y}$ once again represents the concatenated basis function coefficients of the electric and magnetic currents, then the concatenation of (16) and (17), with respect to the testing functions, can be written as

$$
\frac{1}{2} \tilde{\mathbf{I}} \mathbf{y}=\mathbf{B}_{\mathrm{JCM}} \mathbf{y}
$$

where $\mathbf{B}_{\mathrm{JCM}}$ is the discrete operator representing the freespace Green's function operator. If the currents are represented by pulse basis functions and the field is tested by spatially coincidental delta functions in the correct order, the matrix $\tilde{\mathbf{I}}$ is exactly the identity matrix I. For general basis and testing functions, $\tilde{\mathbf{I}}$ is a permuted, banded matrix. A banded matrix can arise when the current is represented by more than one basis function coefficient, e.g., Rao-Wilton-Glisson (RWG) basis functions. Finally, the condition in (18) can be re-arranged as

$$
0=\mathbf{B}_{\mathrm{JCM}}^{\prime} \mathbf{y}
$$

where $\mathbf{B}_{\mathrm{JCM}}^{\prime} \triangleq \mathbf{B}_{\mathrm{JCM}}-\tilde{\mathbf{I}} / 2$. The jump condition can then be implemented as a side constraint in the form of

$$
\min _{\mathbf{y}}\left\{\|\mathbf{A y}-\mathbf{b}\|_{2}^{2}+\alpha_{\mathrm{JCM}}^{2}\left\|\mathbf{B}_{\mathrm{JCM}}^{\prime} \mathbf{y}\right\|_{2}^{2}\right\}
$$

where $\alpha_{\mathrm{JCM}}$ is an appropriate regularization weight. It is apparent that the JCM constraint is in the form of a Tikhonov regularizer.

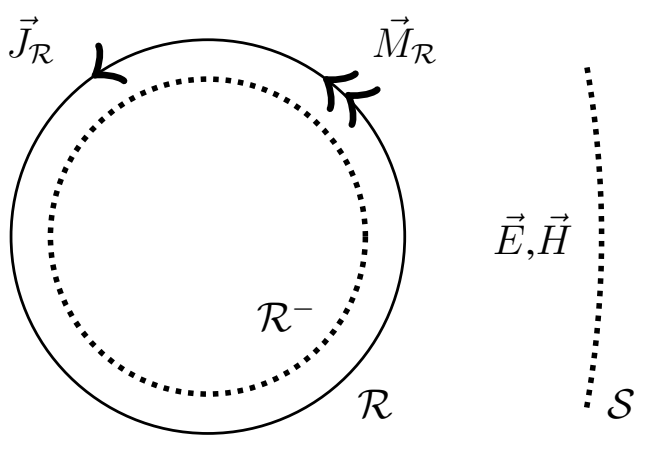

Fig. 2. VPM Method: introduction of virtual measurement points on the surface $\mathcal{R}^{-}$just inside of $\mathcal{R}$. External measurements are made on the surface $\mathcal{S}$. The enforcement of Love's condition on $\mathcal{R}$ is made by setting a zero-field measurement on $\mathcal{R}^{-}$.

\section{B. Virtual Point Method (VPM)}

The second technique, and the one which was proposed in [7], will be referred to as the virtual point method (VPM). This method is an extension of the JCM and its formulation is obtained by evaluating the field integrals on a surface $\mathcal{R}^{-}$ which is inset with respect to $\mathcal{R}$ by an infinitesimal distance. Since the field just inside $\mathcal{R}$ vanishes under Love's condition, (12) and (13) become

$$
\begin{array}{ll}
\overrightarrow{0}=\hat{n} \times\left[-\frac{1}{\eta_{0}} \mathcal{L}\left(\vec{M}_{\mathcal{R}} ; \vec{r}\right)-\mathcal{K}\left(\vec{J}_{\mathcal{R}} ; \vec{r}\right)\right] & \forall \vec{r} \in \mathcal{R}^{-}, \\
\overrightarrow{0}=\hat{n} \times\left[-\eta_{0} \mathcal{L}\left(\vec{J}_{\mathcal{R}} ; \vec{r}\right)+\mathcal{K}\left(\vec{M}_{\mathcal{R}} ; \vec{r}\right)\right] & \forall \vec{r} \in \mathcal{R}^{-},
\end{array}
$$

where we have only considered the tangential field. ${ }^{2}$ Using point testing functions, the discrete form of these equations is formulated like a true measurement on $\mathcal{R}^{-}$, which is why we refer to this method as the virtual (measurement) point method. The setup for this method can be seen in Fig. 2 where we introduce an inner surface $\mathcal{R}^{-}$, upon which the virtual measurement points are placed. After discretizing the equations, the constraint for the VPM will appear as

$$
\mathbf{0}=\mathbf{B}_{\mathrm{VPM}} \mathbf{y}
$$

which is in the same form as the JCM. The side constraint for the VPM can then be incorporated into the formulation as a side constraint in the form of

$$
\min _{\mathbf{y}}\left\{\|\mathbf{A y}-\mathbf{b}\|_{2}^{2}+\alpha_{\mathrm{VPM}}^{2}\left\|\mathbf{B}_{\mathrm{VPM}} \mathbf{y}\right\|_{2}^{2}\right\}
$$

where $\alpha_{\mathrm{VPM}}$ is an appropriate regularization weight.

\section{Dual Surface Method (DSM)}

The dual surface method (DSM) arises from the external analogue to the VPM. In other words, we introduce an evaluation surface $\mathcal{R}^{+}$which is parallel to the source surface $\mathcal{R}$ and offset by an infinitesimal distance. For consistency reasons, the source surface will instead be labeled as $\mathcal{R}^{-}$and the evaluation surface will instead be labeled as $\mathcal{R}$, as shown in Fig. 3. We then evaluate (12) and (13) on $\mathcal{R}$ for the tangential

\footnotetext{
${ }^{2} \mathrm{By}$ the electromagnetic uniqueness theorem, it is sufficient to make the tangential fields zero in order to have a zero internal field.
} 


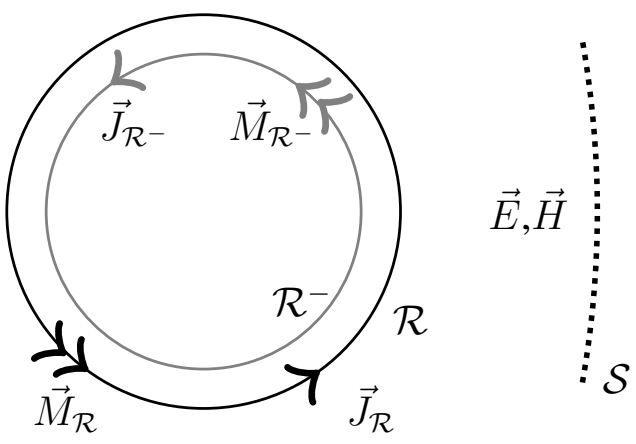

Fig. 3. DSM Method: two equivalent problems for the external field using $\mathcal{R}$ and $\mathcal{R}^{-}$. The $\vec{J}_{\mathcal{R}}$ and $\vec{M}_{\mathcal{R}}$ currents constitute $\mathbf{y}$, and the $\vec{J}_{\mathcal{R}^{-}}$and $\vec{M}_{\mathcal{R}^{-}}$ currents constitute $\mathbf{x}$. The enforcement of Love's condition is made on $\mathcal{R}$ by linking the two sets of currents.

fields thereon as a function of the equivalent currents on $\mathcal{R}^{-}$ (denoted by $\vec{M}_{\mathcal{R}^{-}}$and $\vec{J}_{\mathcal{R}^{-}}$). Thus, we have

$$
\begin{array}{rr}
\hat{n} \times \vec{H}(\vec{r})=\hat{n} \times\left[-\frac{1}{\eta_{0}} \mathcal{L}\left(\vec{M}_{\mathcal{R}^{-}} ; \vec{r}\right)-\mathcal{K}\left(\vec{J}_{\mathcal{R}^{-}} ; \vec{r}\right)\right] & \forall \vec{r} \in \mathcal{R}, \\
\hat{n} \times \vec{E}(\vec{r})=\hat{n} \times\left[-\eta_{0} \mathcal{L}\left(\vec{J}_{\mathcal{R}^{-}} ; \vec{r}\right)+\mathcal{K}\left(\vec{M}_{\mathcal{R}^{-}} ; \vec{r}\right)\right] & \forall \vec{r} \in \mathcal{R} .
\end{array}
$$

Unfortunately, such a relation is not useful as we are not provided with equations to enforce Love's condition on the source surface $\mathcal{R}^{-}$; it is at this point that the formulation of the DSM diverges from the previous two methods.

To begin, we note that the left-hand sides of (25) and (26) correspond to the equivalent currents which would arise from the equivalent problem with respect to $\mathcal{R}$ under Love's condition (hence the use of $\mathcal{R}$ for the evaluation surface). Thus, the next step is to introduce a separate equivalent problem for $\mathcal{R}$, where we introduce equivalent currents $\vec{J}_{\mathcal{R}}$ and $\vec{M}_{\mathcal{R}}$, as shown in Fig. 3. Under Love's condition, these equivalent currents will be related to the tangential fields, and thus they can be substituted into (25) and (26) as

$$
\vec{J}_{\mathcal{R}}(\vec{r})=\hat{n} \times\left[-\frac{1}{\eta_{0}} \mathcal{L}\left(\vec{M}_{\mathcal{R}^{-}} ; \vec{r}\right)-\mathcal{K}\left(\vec{J}_{\mathcal{R}^{-}} ; \vec{r}\right)\right] \quad \forall \vec{r} \in \mathcal{R},
$$

$$
\vec{M}_{\mathcal{R}}(\vec{r})=-\hat{n} \times\left[-\eta_{0} \mathcal{L}\left(\vec{J}_{\mathcal{R}^{-}} ; \vec{r}\right)+\mathcal{K}\left(\vec{M}_{\mathcal{R}^{-}} ; \vec{r}\right)\right] \quad \forall \vec{r} \in \mathcal{R} .
$$

If we allow $\mathbf{x}$ to be the concatenated vector of basis function coefficients for the electric and magnetic currents on $\mathcal{R}^{-}$, then the above relations can be discretized into

$$
\tilde{\mathbf{I}} \mathbf{y}=\mathbf{B}_{\mathrm{DSM}} \mathbf{x},
$$

where $\tilde{\mathbf{I}}$ is once again a permuted, banded matrix as in (18), and $\mathbf{B}_{\mathrm{DSM}}$ represents the discrete form of the right-hand sides of (27) and (28). The complete cost functional to be minimized takes the form

$$
\min _{\mathbf{x}, \mathbf{y}}\{\underbrace{\left\|\mathbf{A}_{\mathbf{x}} \mathbf{x}-\mathbf{b}\right\|_{2}^{2}}_{\text {data misfit for } \mathbf{x}}+\underbrace{\left\|\mathbf{A}_{\mathbf{y}} \mathbf{y}-\mathbf{b}\right\|_{2}^{2}}_{\text {data misfit for } \mathbf{y}}+\alpha_{\text {DSM }}^{2} \underbrace{\left\|\mathbf{B}_{\mathrm{DSM}} \mathbf{x}-\tilde{\mathbf{I}} \mathbf{y}\right\|_{2}^{2}}_{\text {Love's cond. for } \mathbf{y}}\},
$$

where $\mathbf{A}_{\mathbf{y}}=\mathbf{A}$ as in the previous methods, $\mathbf{A}_{\mathbf{x}}$ is the equivalent of $\mathbf{A}$ for $\mathbf{x}$, and $\alpha_{\mathrm{DSM}}$ is an appropriate regularization weight.

\section{Analysis of Methods}

We would now like to analyze and compare the three methods in order to predict similarities and/or differences. We will consider an ideal numerical case and a practical numerical case.

\section{A. Ideal Case}

In the ideal case, the currents are finely discretized, and the auxiliary surfaces $\mathcal{R}^{-}$for the VPM and DSM are at an insignificant distance from $\mathcal{R}$. As a consequence, the field at almost any location suffers from insignificant approximation error. In Appendix A, it can be shown that under certain assumptions, the product terms of the JCM constraint in (18) may be written as

$$
\begin{gathered}
\tilde{\mathbf{I}} \rightarrow \boldsymbol{\psi}_{2}-\boldsymbol{\psi}_{1}, \\
\mathbf{B}_{\mathrm{JCM}} \mathbf{y} \rightarrow \frac{\boldsymbol{\psi}_{2}+\boldsymbol{\psi}_{1}}{2} .
\end{gathered}
$$

The vectors $\psi_{1}$ and $\psi_{2}$ are vectors of scalar coefficients which represent the tangential inner fields $\left(\psi_{1}\right), \hat{n} \times \vec{E}_{1}$ and $\hat{n} \times \vec{H}_{1}$, and the tangential outer fields $\left(\psi_{2}\right), \hat{n} \times \vec{E}_{2}$ and $\hat{n} \times \vec{H}_{2}$, which are a result of the MoM procedure. The usefulness of this representation is the intuitive understanding that it provides while being mathematically consistent (see Appendix A). Using this vector field representation, we can evaluate (18) with the vector fields as

$$
\frac{\boldsymbol{\psi}_{2}-\boldsymbol{\psi}_{1}}{2}=\frac{\boldsymbol{\psi}_{2}+\boldsymbol{\psi}_{1}}{2} \Longrightarrow \mathbf{0}=\boldsymbol{\psi}_{1} .
$$

That is, by satisfying the JCM relation, we are effectively setting the inner tangential fields to zero, which results in a zero internal field. The procedure in Appendix A can be repeated for the VPM and DSM methods to obtain the other side constraint products, namely

$$
\begin{aligned}
& \mathbf{B}_{\mathrm{VPM}} \mathbf{y} \rightarrow \boldsymbol{\psi}_{1}, \\
& \mathbf{B}_{\mathrm{DSM}} \mathbf{x} \rightarrow \boldsymbol{\psi}_{2},
\end{aligned}
$$

which follows from the general equivalence principle. Thus, the VPM side constraint in (23) enforces

$$
\mathbf{0}=\psi_{1},
$$

and the DSM side constraint in (29) enforces

$$
\boldsymbol{\psi}_{2}-\boldsymbol{\psi}_{1}=\boldsymbol{\psi}_{2}, \Longrightarrow \mathbf{0}=\boldsymbol{\psi}_{1} .
$$

Considering (33), (36), and (37), all three of the methods appear to provide an identical enforcement of a zero internal field. In fact, we can show that the side constraint operators are equivalent. To this end, by noting (31) and (34) we can write (32) as

$$
\begin{gathered}
\mathbf{B}_{\mathrm{JCM}} \mathbf{y}=\frac{\overbrace{\mathbf{I} \mathbf{y}+\mathbf{B}_{\mathrm{VPM}} \mathbf{y}}^{\psi_{2}}+\overbrace{\mathbf{B}_{\mathrm{VPM}} \mathbf{y}}^{\psi_{1}}=\frac{1}{2} \tilde{\mathbf{I}} \mathbf{y}+\mathbf{B}_{\mathrm{VPM}} \mathbf{y},}{\mathbf{B}_{\mathrm{JCM}} \mathbf{y}-\frac{1}{2} \tilde{\mathbf{I}} \mathbf{y}=\mathbf{B}_{\mathrm{VPM}} \mathbf{y} \Longrightarrow \mathbf{B}_{\mathrm{JCM}}^{\prime} \mathbf{y}=\mathbf{B}_{\mathrm{VPM}} \mathbf{y} .}
\end{gathered}
$$


This shows the equivalency of the side constraints in (20) and (24). A similar equivalency can be made for the DSM by recognizing that we can approximate $\mathbf{x} \approx \mathbf{y}$, since $\mathcal{R}^{-} \rightarrow \mathcal{R}$; thus, the equivalent operator $\left(-\tilde{\mathbf{I}}+\mathbf{B}_{\mathrm{DSM}}\right)$ from (29) can be rearranged into the JCM or VPM constraint using the procedure above.

Using the vector field representation of the Love's constraint (i.e., $\mathbf{0}=\boldsymbol{\psi}_{1}$ ), it is possible to obtain the solution form which satisfies this constraint. To begin, we will assume that $\tilde{\mathbf{I}} \rightarrow \mathbf{I}$. Thus, the product $\tilde{\mathbf{I}} \mathbf{y}$ also represents the unknown vector, i.e.,

$$
\mathbf{y}=\psi_{2}-\psi_{1}
$$

Note that in this case, $\mathbf{y}$ represents an unconstrained solution, on account of the $\psi_{1}$ component which should not be present in the Love's currents. To obtain the solution which satisfies the Love's constraint, we simply substitute the field vector representation of the Love's constraint from (33), (36), or (37), into (39):

$$
\mathbf{y}=\psi_{2}, \quad \text { (ideal solution). }
$$

As expected, the Love's constraint favors a solution which does not produce an internal field. It should be noted that $\boldsymbol{\psi}_{2}$ is not necessarily the desired solution; undesired nonradiating currents may still exist, as described in Section III. In order to remove these currents, we require some additional regularization; however, this is made much easier by the removal of a large portion of non-radiating currents.

For the ideal case, we have shown that the methods for enforcing Love's condition appear identical. However, the ideal case is unlikely to occur in practice, as we likely cannot or do not want to have a finely discretized problem. ${ }^{3}$ As a result, the field in the vicinity of the currents will suffer from increased approximation errors due to the singular nature of the Green's function. Additional concessions must be made to address this, which can result in tangible differences between the three methods.

\section{B. Practical Case}

In the practical case, the singularity of the Green's function must be accounted for in some manner. For the JCM, the only option is to incorporate singularity extraction or cancellation techniques, which are commonly used in forward scattering problems [26]. For the VPM and DSM, there is also the option of increasing the separation distance between $\mathcal{R}$ and the auxiliary surface $\mathcal{R}^{-}$, which avoids the calculation of nearsingular fields. Hereinafter, it will be assumed that an increased separation distance is used for the VPM and DSM. The VPM has historically been used this way [7], [9], whereas the DSM will see some unique advantages.

Given this assumption, we can re-examine the side constraints while accounting for any changes. In particular, the outputs of the side constraint operators are potentially different. For the JCM, if we assume that the singularity extraction is done well, both the constraint and the solution under the

\footnotetext{
${ }^{3}$ Aside from computational requirements, a finely discretized problem is more susceptible to instability, as the solution space allows for higher spatial frequencies which correspond to smaller singular values [25].
}

vector field representation are effectively the same as in the ideal case.

For the VPM, the output of $\mathbf{B}_{\mathrm{VPM}} \mathbf{y}$ is no longer exactly $\psi_{1}$. Indeed, by the smoothing properties [14] of the Green's function, the output is highly unlikely to be precisely the same at the new surface. The displacement of $\mathcal{R}^{-}$has been shown to be equivalent to the spectral filtering of the Green's function [9], [27], which avoids the singularity of the Green's function. However, this remains an approximation error and can become noticeable in the inverse source problem. We will denote the approximated field by $\boldsymbol{\psi}_{1}^{\prime}$, i.e.,

$$
\mathbf{B}_{\mathrm{VPM}} \mathbf{y} \rightarrow \boldsymbol{\psi}_{1}^{\prime}, \quad \text { (practical case). }
$$

This new output can be substituted into the VPM side constraint in (23) to obtain the practical constraint, $\mathbf{0}=\boldsymbol{\psi}_{1}^{\prime}$. It will be convenient to define $\Delta \psi_{1}$, the difference between the exact and approximated inner field, as $\Delta \boldsymbol{\psi}_{1} \triangleq \boldsymbol{\psi}_{1}-\boldsymbol{\psi}_{1}^{\prime}$. Thus, the VPM constraint (23) can also be written as

$$
\mathbf{0}=\boldsymbol{\psi}_{1}-\Delta \boldsymbol{\psi}_{1}, \quad \text { (practical constraint, VPM). }
$$

In this case, the inner field $\psi_{1}$ is not necessarily zero; it can be equal to $\Delta \psi_{1}$ by (42). Using the same procedure as in the ideal case, we can obtain the practical solution form for the VPM. Substituting (42) into (39) results in

$$
\mathbf{y}=\boldsymbol{\psi}_{2}-\Delta \boldsymbol{\psi}_{1}, \quad \text { (practical solution, VPM). }
$$

Thus, the VPM can suffer from increased error in the form of $\Delta \psi_{1}$, which increases with the separation distance (i.e., this error vanishes as $\mathcal{R}^{-} \rightarrow \mathcal{R}$ ). If the data equation is otherwise satisfied, which requires $\boldsymbol{\psi}_{2}$ to contain the radiating currents, then the $\mathbf{y}=-\Delta \psi_{1}$ currents are necessarily non-radiating currents with respect to $\mathcal{S}$. Note that these currents produce a non-zero internal field in the region between $\mathcal{R}$ and $\mathcal{R}^{-}$only; the field is effectively zero elsewhere in $\mathcal{D}_{1}$. The presence of this non-zero internal field will be demonstrated in Section VI.

For the DSM, we would initially expect the output of $\mathbf{B}_{\mathrm{DSM}} \mathbf{x}$ to remain the same, as there is no apparent approximation involved. However, we will propose that the output can be different, which will later be supported by simulation results. First, note that the desired or true Love's currents on $\mathcal{R}^{-}$ and $\mathcal{R}$ will satisfy the DSM constraint. Thus, any undesired non-radiating currents, even those contained in $\psi_{2}$, can only be reconstructed if the corresponding currents $\mathbf{x}_{\mathrm{NR}}$ such that $\mathbf{B}_{\mathrm{DSM}} \mathbf{x}_{\mathrm{NR}}=\mathbf{y}_{\mathrm{NR}}$ are simultaneously reconstructed during the optimization process. If only one of $\mathbf{x}_{\mathrm{NR}}$ or $\mathbf{y}_{\mathrm{NR}}$ currents exist at a particular iteration of the reconstruction algorithm, it is likely that this breaks the DSM constraint. Thus, there is a reasonable chance that the currents are removed at the next iteration. This is not guaranteed, i.e., the other $\mathbf{x}_{\mathrm{NR}}$ or $\mathbf{y}_{\mathrm{NR}}$ currents may instead be introduced to the solution, but we propose that the restriction on the non-radiating currents is not unlikely and represents an advantage for the DSM.

Under this proposition, the output of $\mathbf{B}_{\mathrm{DSM}} \mathbf{x}$ may differ from $\boldsymbol{\psi}_{2}$. Denoting the restricted field as $\boldsymbol{\psi}_{2}^{\prime}$, we obtain the practical constraint by changing the right-hand-side in (29):

$$
\begin{gathered}
\boldsymbol{\psi}_{2}-\boldsymbol{\psi}_{1}=\boldsymbol{\psi}_{2}^{\prime}, \\
\left.\mathbf{0}=\boldsymbol{\psi}_{1}-\Delta \boldsymbol{\psi}_{2}, \quad \text { (practical constraint, DSM }\right),
\end{gathered}
$$


where $\Delta \psi_{2} \triangleq \psi_{2}-\psi_{2}^{\prime}$. Satisfying this constraint results in a solution of the form

$$
\mathbf{y}=\boldsymbol{\psi}_{2}-\Delta \boldsymbol{\psi}_{2}=\boldsymbol{\psi}_{2}^{\prime}, \quad \text { (practical solution, DSM), }
$$

where (44) has been substituted into (39).

It is important to reinforce that the DSM is not guaranteed to provide a greater restriction; for instance, it is entirely possible that the same non-radiating currents in the JCM solution are also present in the DSM solution. We simply propose that this occurrence is unlikely, and that the DSM solution will generally be more restricted.

\section{Projection Methods}

Due to the form of the JCM and DSM constraints, these methods are readily transformed into so-called projection methods [10], [11]. Instead of using a side constraint, we simply operate on the total currents to remove the portion which produces a non-zero internal field. To illustrate, consider that the JCM constraint may be written as

$$
\frac{1}{2} \tilde{\mathbf{I}} \mathbf{y}=\mathbf{B}_{\mathrm{JCM}} \mathbf{y} \Longrightarrow \tilde{\mathbf{I}} \mathbf{y}=\frac{1}{2} \tilde{\mathbf{I}} \mathbf{y}+\mathbf{B}_{\mathrm{JCM}} \mathbf{y}
$$

Employing the vector field representation, we then have

$$
\left(\frac{1}{2} \tilde{\mathbf{I}}+\mathbf{B}_{\mathrm{JCM}}\right) \mathbf{y} \rightarrow \frac{\boldsymbol{\psi}_{2}-\boldsymbol{\psi}_{1}}{2}+\frac{\boldsymbol{\psi}_{2}+\boldsymbol{\psi}_{1}}{2}=\boldsymbol{\psi}_{2}
$$

Thus, the combined operator $\tilde{\mathbf{I}} / 2+\mathbf{B}_{\mathrm{JCM}}$ has the effect of projecting arbitrary currents onto the Love's currents. In order to obtain the actual basis function coefficients in $\mathbf{y}$, we require the additional operator $\tilde{\mathbf{I}}^{-1}$. For non-square matrices, this represents a Moore-Penrose pseudo-inverse; if $\tilde{\mathbf{I}}$ is square, we require the standard matrix inverse. If we use the same basis and testing functions, $\tilde{\mathbf{I}}$ becomes a square Gram matrix whose inverse is readily computed [10]. The total projection operator $\mathbf{P}=\tilde{\mathbf{I}}^{-1}\left(\tilde{\mathbf{I}} / 2+\mathbf{B}_{\mathrm{JCM}}\right)$ can be incorporated as a preconditioner or used on the solution to the unconstrained problem. This technique for the JCM was first proposed in [10]. Note that this technique will only remove the currents which produce a non-zero internal field; we lose the inherent relaxation of the data equation which occurs with the side constraint. As a consequence, the projection method may require greater additional regularization, which can be difficult for the reasons outlined in Section III.

The projection method can also be applied to the DSM by re-arranging the constraint as

$$
\mathbf{y}=\mathbf{P x}=\tilde{\mathbf{I}}^{-1} \mathbf{B}_{\mathrm{DSM}} \mathbf{x} .
$$

Note that in this case, the $\mathbf{y}$ currents are completely decoupled from their associated data equation $\left(\mathbf{A}_{\mathbf{y}} \mathbf{y}=\mathbf{b}\right)$, as they are exactly defined through the projection operator. This is similar to existing methods which obtain the Love's currents by propagating the unconstrained currents [9], [11].

\section{Summary}

Finally, we would like to summarize the discussion in this section. To begin, we reiterate the purpose of the Love's constraint: to remove the undesired non-radiating currents of the problem. The goal of this section was to examine the relative effectiveness of the three methods for formulating the constraint. To this end, the methods were generalized and then analyzed in ideal and practical cases. A summary of this analysis, which includes the ideal and practical solution forms, can be found in Table I.

By the practical solution forms, it was possible to infer potential differences between the three methods. Using the JCM as the default method, the VPM was found to have a lesser restriction on non-radiating currents, and the DSM was suggested to have a greater restriction. Although this does not guarantee any differences, in general the degree of restriction will likely determine the level and difficulty of the additional regularization, as the total set of undesired nonradiating currents has been reduced. Thus, in many cases the degree of restriction will also dictate the potential accuracy of the reconstructed solution.

Finally, we would like to emphasize that each method has its own advantages and disadvantages. Although the VPM has the weakest side constraint, it is the easiest to implement. In contrast, the DSM has the strongest side constraint, but requires a larger computational cost. In addition, its reconstructed currents cannot be made to fit around the AUT as tightly as the other methods. Lastly, the JCM is more difficult to implement, as we must address the singularity of the Green's function, but is computationally efficient when used in the projection form.

\section{RESUlTS}

In order to verify and evaluate the relative performance of the available methods for enforcing Love's condition, we will examine two examples: an infinitesimal dipole, and a circular horn antenna. The first example will be examined more closely in order to demonstrate the findings in this paper. The second example represents a practical case which is more briefly examined to reinforce the previous findings.

\section{A. Formulation and Implementation Details}

The data operators and Love's constraints were constructed through a MoM procedure [28] using RWG basis functions [28] and point testing functions. In addition, the operators have been scaled so that the relative magnitudes of the electric and magnetic currents are similar [9]. For the JCM, the singularity of the Green's function was cancelled by using a height-independent radial-angular- $R^{2}$ singularity cancellation technique [26]. The additional regularization for the Love's methods was implemented in the form of Krylov subspace regularization [19]. All regularization weights were chosen in an ad hoc way to minimize the solution error; i.e., the reconstructed currents were compared to the true Love's currents and the regularization weights were adjusted accordingly. This is done because the selection of multiple regularization weights is not trivial, but note that this limitation is shared by all the regular Love's methods. The measured data consists of the magnitude and phase of the electric field. 
TABLE I

SUMMARY OF METHODS

\begin{tabular}{|c||c||c||c||c||c|}
\hline Method & Cost Functional & Ideal Constraint & Ideal Solution & Practical Constraint & Practical Solution \\
\hline $\mathrm{JCM}$ & $\|\mathbf{A} \mathbf{y}-\mathbf{b}\|_{2}^{2}+\alpha_{\mathrm{JCM}}^{2}\left\|\mathbf{B}_{\mathrm{JCM}}^{\prime} \mathbf{y}\right\|_{2}^{2}$ & $\boldsymbol{\psi}_{1}=\mathbf{0}$ & $\boldsymbol{\psi}_{2}$ & $\boldsymbol{\psi}_{1}=\mathbf{0}$ & $\boldsymbol{\psi}_{2}$ \\
\hline $\mathrm{VPM}$ & $\|\mathbf{A} \mathbf{y}-\mathbf{b}\|_{2}^{2}+\alpha_{\mathrm{VPM}}^{2}\left\|\mathbf{B}_{\mathrm{VPM}} \mathbf{y}\right\|_{2}^{2}$ & $\boldsymbol{\psi}_{1}=\mathbf{0}$ & $\boldsymbol{\psi}_{2}$ & $\boldsymbol{\psi}_{1}-\Delta \boldsymbol{\psi}_{1}=\mathbf{0}$ & $\boldsymbol{\psi}_{2}-\Delta \boldsymbol{\psi}_{1}$ \\
\hline $\mathrm{DSM}$ & $\left\|\mathbf{A}_{\mathbf{y}} \mathbf{y}-\mathbf{b}\right\|_{2}^{2}+\left\|\mathbf{A}_{\mathbf{x}} \mathbf{x}-\mathbf{b}\right\|_{2}^{2}+\alpha_{\mathrm{DSM}}^{2}\left\|\mathbf{B}_{\mathrm{DSM}} \mathbf{x}-\tilde{\mathbf{I}} \mathbf{y}\right\|_{2}^{2}$ & $\boldsymbol{\psi}_{1}=\mathbf{0}$ & $\boldsymbol{\psi}_{2}$ & $\boldsymbol{\psi}_{1}-\Delta \boldsymbol{\psi}_{2}=\mathbf{0}$ & $\boldsymbol{\psi}_{2}^{\prime}$ \\
\hline
\end{tabular}

\section{B. Infinitesimal Dipole Antenna - Full-View Data}

The first antenna to be analyzed is an infinitesimal dipole antenna at the origin, oriented along the $z$-axis, and radiating at a frequency of $0.9 \mathrm{GHz}$. The measured data were obtained from analytical formulas [13, Ch. 4]. The main reconstruction surface $\mathcal{R}$ is a sphere of radius $r_{0}=10 \mathrm{~cm}$, or $r_{0} \sim \lambda / 3$. The maximum mesh element size was selected as $l_{e}=\lambda / 10$. The auxiliary surface for the VPM is a sphere of radius $r_{\mathrm{VPM}}^{\prime}=$ $r_{0}-l_{e}$, and the auxiliary surface for the DSM is a sphere of radius $r_{\mathrm{DSM}}^{\prime}=5 \mathrm{~cm} \sim r_{0}-\lambda / 5 .{ }^{4}$ The number of observation points ${ }^{5}$ on the auxiliary surface for the VPM is the same as the number of elements on $\mathcal{R}$. The point testing locations for the JCM and DSM are at the center of mesh elements; thus, the number of rows for each side constraint is the same. Noise has not been added to the measured data, although the data operator suffers from approximation error. The measurement surface $\mathcal{S}$ is a sphere of radius $r_{\mathcal{S}}=20 \mathrm{~cm} \sim 2 \lambda / 3$. For the first set of results, the measurements were distributed over $\mathcal{S}$ with angular steps of $\Delta \theta=\Delta \varphi=10^{\circ}$.

A set of results for this scenario are shown in Fig. 4. The plots on the left-hand side show the electric field magnitude on $\mathcal{R}$, and those on the right-hand side show the magnetic field magnitude. ${ }^{6}$ The field sampling is at the mesh element centroid, and the field values are obtained from the currents by using (2). The true fields are shown in Fig. 4(a) and (b). Following this, the reconstructed fields over $\mathcal{R}$ are shown for:

1) an identity Tikhonov constraint in Fig. 4(c) and (d),

2) the JCM method in Fig. 4(e) and (f),

3) the VPM method in Fig. 4(g) and (h),

4) the DSM method in Fig. 4(i) and (j).

In addition, error values for the reconstructed fields, as well as the reconstructed far-field, are given in Table II. In addition to the four methods in Fig. 4, the projection methods for the JCM and DSM are included, denoted by "-P". The field error quantities are defined as

$$
\epsilon \triangleq\left\|\Psi^{\text {true }}-\Psi^{\text {rec }}\right\|_{2} /\left\|\Psi^{\text {true }}\right\|_{2}
$$

where the superscript "true" denotes the analytical field, "rec" denotes the reconstructed field, and $\boldsymbol{\Psi}$ is the electric field $\left(\epsilon_{E}\right.$ and $\left.\epsilon_{\mathrm{FF}}\right)$ or magnetic field $\left(\epsilon_{H}\right)$. The quantities $\epsilon_{E}$ and $\epsilon_{H}$ are referred to the field at the element centroids on $\mathcal{R}$ (for

\footnotetext{
${ }^{4}$ For the VPM, the separation distance should be minimized while avoiding the singularity of the Green's function, which is why the separation distance is chosen as the element size [7]. For the DSM, the separation distance should be larger; see Section VI-D.

${ }^{5}$ Each observation point represents four equations, as there are two tangential delta functions and two field components $(\vec{E}$ and $\vec{H})$.

${ }^{6}$ The phase error is implicitly given by the field errors in Table II.
}

TABLE II

RECONSTRUCTION ERRORS FOR THE FULL-VIEW INFINITESIMAL DIPOLE.

\begin{tabular}{|c||c||c||c|}
\hline Method & $\epsilon_{E}$ & $\epsilon_{H}$ & $\epsilon_{\mathrm{FF}}$ \\
\hline \hline Identity Tikhonov & $63 \%$ & $74 \%$ & $0.058 \%$ \\
\hline JCM & $7.2 \%$ & $4.0 \%$ & $0.080 \%$ \\
\hline JCM-P & $13 \%$ & $9.2 \%$ & $0.082 \%$ \\
\hline VPM & $9.0 \%$ & $7.8 \%$ & $0.088 \%$ \\
\hline DSM & $1.1 \%$ & $0.68 \%$ & $0.080 \%$ \\
\hline DSM-P & $2.6 \%$ & $1.7 \%$ & $0.51 \%$ \\
\hline
\end{tabular}

these quantities, the magnitude and phase is included in $\Psi$ ). The quantity $\epsilon_{\mathrm{FF}}$ is referred to a far-field sphere $\left(r_{\mathrm{FF}} \sim 10 \lambda\right)$ with sampling points spaced by $\Delta \theta=\Delta \phi=20^{\circ}$. In addition, only the normalized magnitude is considered in $\epsilon_{\mathrm{FF}}$. Given the freedom in defining the errors, it must be emphasized that these errors should not be interpreted as a formal analysis of the individual methods. We are instead concerned with the relative differences between the methods.

The identity Tikhonov constraint ${ }^{7}$ is included in the results to demonstrate the usefulness of enforcing Love's condition. Referring to Fig. 4(c) and (d), as well as the reported $\epsilon_{E}$ and $\epsilon_{H}$ in Table II, we see that there is a relatively large magnitude and phase error in the reconstructed fields on $\mathcal{R}$ when using the identity Tikhonov regularizer. This error is due to the inclusion of $\boldsymbol{\psi}_{1}$ components in the solution, i.e., undesired non-radiating currents. However, the inclusion of fewer constraints allows for a smaller data misfit cost functional; thus, as a consequence of the uniqueness principle, the far-field error $\epsilon_{\mathrm{FF}}$ is the lowest of any method. Note that this is entirely dependent on using accurate, well-sampled data over a closed surface $\mathcal{S}$.

For the three methods of enforcing Love's condition, the general accuracy of the reconstructed field on $\mathcal{R}$ follows the expected restriction on the non-radiating currents for the constrained problem; see $\epsilon_{E}$ and $\epsilon_{H}$ in Table II. Of the three main methods, the DSM is the most accurate, and the VPM is the least accurate. We can also see this effect visually by noting Fig. 4(i)-(j) where the DSM reconstruction is noticeably closer to the true solution. In addition, the VPM appears the least smooth and is the most similar to the identity Tikhonov. This indicates that there is a non-trivial $\boldsymbol{\psi}_{1}$ portion to the VPM solution, which was anticipated by the analysis in Section V. This can be verified by employing the JCM operators to extract

\footnotetext{
${ }^{7}$ The cost functional to be minimized is $\min _{\mathbf{y}}\left\{\left\|\mathbf{A}_{\mathbf{y}} \mathbf{y}-\mathbf{b}\right\|_{2}^{2}+\alpha^{2}\|\mathbf{y}\|_{2}^{2}\right\}$.
} 


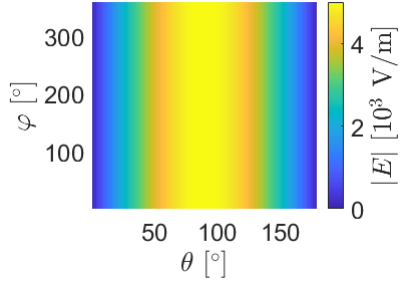

(a) $\left|\vec{E}^{\text {true }}\right|$

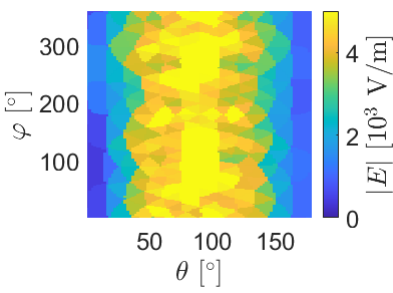

(c) $\left|\vec{E}^{\text {rec }}\right|$, Tikhonov

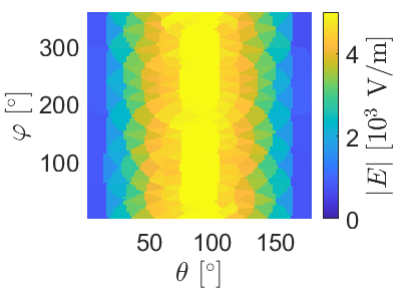

(e) $\left|\vec{E}^{\text {rec }}\right|$, JCM

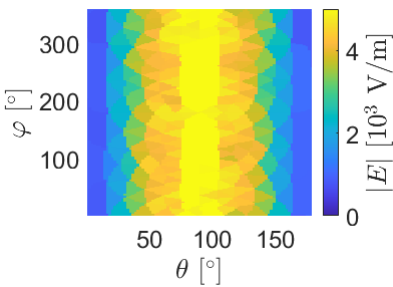

(g) $\left|\vec{E}^{\text {rec }}\right|$, VPM

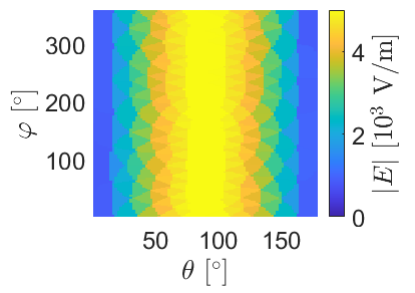

(i) $\left|\vec{E}^{\mathrm{rec}}\right|$, DSM

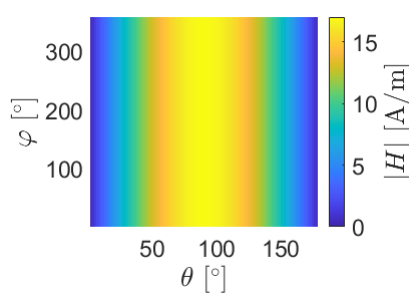

(b) $\left|\vec{H}^{\text {true }}\right|$

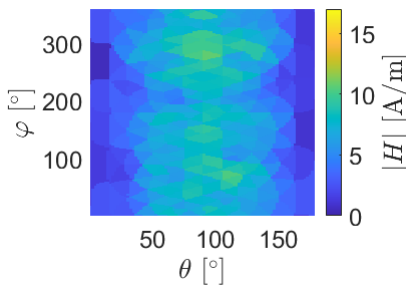

(d) $\left|\vec{H}^{\text {rec }}\right|$, Tikhonov

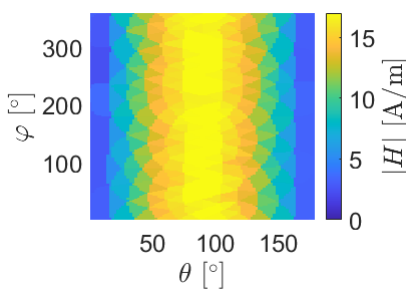

(f) $\left|\vec{H}^{\text {rec }}\right|$, JCM

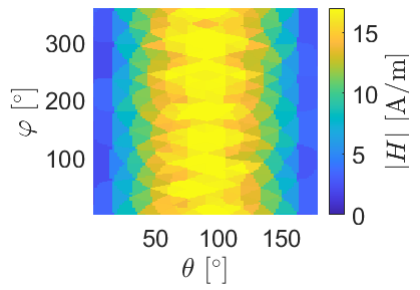

(h) $\left|\vec{H}^{\text {rec }}\right|$, VPM

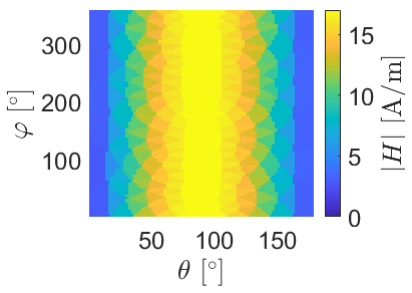

(j) $\left|\vec{H}^{\text {rec }}\right|$, DSM
Fig. 4. True and reconstructed field magnitudes on $\mathcal{R}$ for the infinitesimal dipole problem with full-view data.

$\psi_{1}$ and $\psi_{2}$ by

$$
\boldsymbol{\psi}_{1}=\mathbf{B}_{\mathrm{JCM}}^{\prime} \mathbf{y}, \quad \boldsymbol{\psi}_{2}=\mathbf{B}_{\mathrm{JCM}} \mathbf{y}+\frac{\tilde{\mathbf{I}}}{2} \mathbf{y}
$$

The norms of each field vector for the three methods, as well as the product $\tilde{\mathbf{I}} \mathbf{y}$, are shown in Table III. There is a significantly larger presence of $\psi_{1}$ in the VPM solution, which was predicted in Section V; this results in a larger error for the VPM. Although this inner field portion is large for the VPM, it
TABLE III

FIELD VECTOR NORMS FOR EACH OF THE MAIN METHODS FOR THE FULL-VIEW INFINITESIMAL DIPOLE.

\begin{tabular}{|c||c||c||c|}
\hline Method & $\left\|\boldsymbol{\psi}_{2}\right\|_{2}$ & $\left\|\boldsymbol{\psi}_{1}\right\|_{2}$ & $\|\tilde{\mathbf{I}}\|_{2}$ \\
\hline \hline JCM & 321 & 9 & 327 \\
\hline VPM & 320 & 36 & 325 \\
\hline DSM & 319 & 10 & 326 \\
\hline
\end{tabular}

is relatively small compared to the outer field $\boldsymbol{\psi}_{2}$, which is why the VPM still produces a reasonable solution. The norm of the İy product has been included to show that each solution has been evenly regularized from a minimum-norm perspective. Thus, we can reasonably infer that the relative differences in accuracy between the methods are due to their restriction on the undesired non-radiating currents. When the restriction is greater, the subsequent Krylov subspace regularization can more easily produce an accurate solution.

Regardless of the relative accuracy of the reconstructed fields on $\mathcal{R}$, the resulting far-fields are very accurate (see $\epsilon_{\mathrm{FF}}$ in Table II). This is to be expected, as most of the nonradiating currents which affect the reconstruction accuracy are by definition non-radiating with respect to the far-field. As described in Section II, this only occurs because the measured data are well-sampled over a closed $\mathcal{S}$. A case with insufficient or poorly-sampled measured data will be examined shortly.

In addition to the three constrained methods, the projection methods for the VPM and DSM were examined. In general, the reconstruction accuracy suffered with respect to the constrained method. This was predicted in Section V, where it was noted that it would be more difficult to further regularize the problem, which appears to be the case here. However, the projection methods come with the advantage of not requiring a weight for the Love's constraint, which is useful for a practical problem with no a priori knowledge of the solution. We also note the relatively large far-field error in Table II for the DSM$\mathrm{P}$, which is likely due to the decoupling of the measured data and $\mathbf{y}$; i.e., $\mathbf{y}$ for the DSM-P does not satisfy the measured data as well as other methods.

\section{Infinitesimal Dipole Antenna - Sectoral-View Data}

We would now like to look at a case with poorly-sampled data. To this end, the measured data were taken from a sector on $\mathcal{S}$ defined as $0<\theta<50^{\circ}$ and $0<\varphi<90^{\circ}$, using the same sampling density as used previously. By the uniqueness theorem, this sector of data is insufficient in order to uniquely define the far-field; thus, the reconstructed currents will be free to produce arbitrary fields in other regions. In other words, there will be additional non-radiating currents, defined with respect to $\mathcal{S}$, in this problem.

The reconstructed field magnitudes on $\mathcal{R}$ are shown in Fig. 5, and the field error values are shown in Table IV. In addition, the reconstructed far-field electric field magnitudes are shown on a $\mathrm{dB}$ scale in Fig. 6. Unlike the first case, there is a larger discrepancy between the three methods. In particular, the DSM remains very accurate, whereas the VPM and JCM suffer greatly; this applies to both the fields on $\mathcal{R}$ 


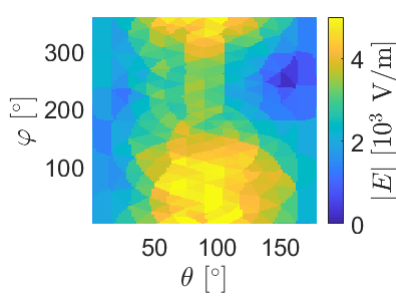

(a) $\left|\vec{E}^{\mathrm{rec}}\right|$, JCM

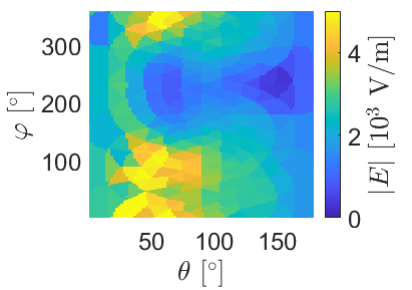

(c) $\left|\vec{E}^{\mathrm{rec}}\right|$, VPM

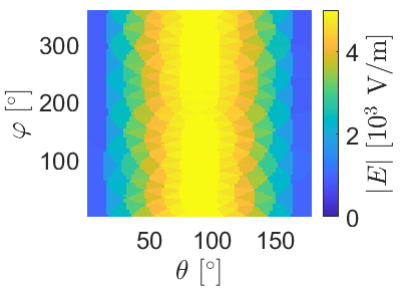

(e) $\left|\vec{E}^{\text {rec }}\right|$, DSM

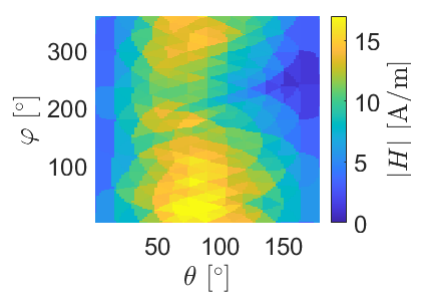

(b) $\left|\vec{H}^{\text {rec }}\right|$, JCM

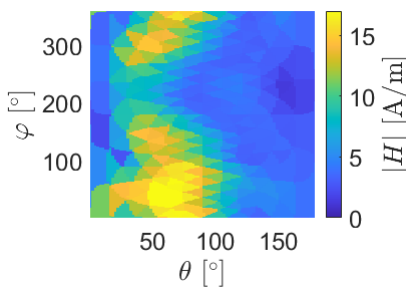

(d) $\left|\vec{H}^{\text {rec }}\right|$, VPM

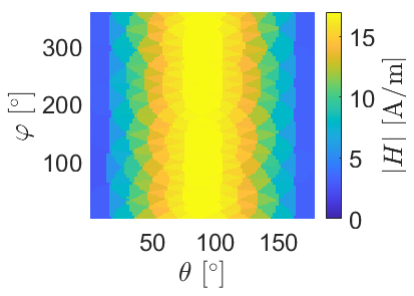

(f) $\left|\vec{H}^{\text {rec }}\right|$, DSM (g) $\left|\vec{E}^{\mathrm{rec}}\right|$, DSM-P

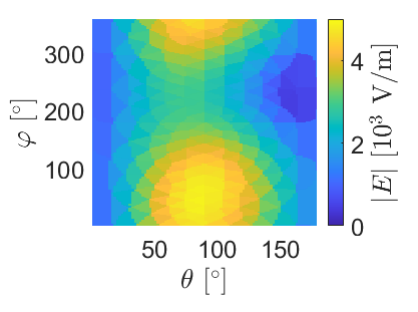

(g)

Fig. 5. Reconstructed field magnitudes on $\mathcal{R}$ for the infinitesimal dipole problem with sectoral-view data.

TABLE IV

RECONSTRUCTION ERRORS FOR THE SECTORAL-VIEW INF. DIPOLE.

\begin{tabular}{|c||c||c||c|}
\hline Method & $\epsilon_{E}$ & $\epsilon_{H}$ & $\epsilon_{\mathrm{FF}}$ \\
\hline \hline Tikhonov & $78 \%$ & $91 \%$ & $50 \%$ \\
\hline JCM & $39 \%$ & $34 \%$ & $27 \%$ \\
\hline JCM-P & $66 \%$ & $63 \%$ & $49 \%$ \\
\hline VPM & $75 \%$ & $71 \%$ & $51 \%$ \\
\hline DSM & $2.8 \%$ & $2.1 \%$ & $1.3 \%$ \\
\hline DSM-P & $33 \%$ & $32 \%$ & $26 \%$ \\
\hline
\end{tabular}

and the far-field. By the analysis in Section V, it is reasonable to assume that the DSM constraint does indeed provide a greater restriction on the set of non-radiating currents, which has been enlarged by the additional non-radiating currents. Incidentally, the nature of these currents can be visualized by the reconstructed field magnitudes in Fig. 5; it can be inferred that these currents partly cancel the true Love's currents. Equivalently, a large portion of the desired solution is non-

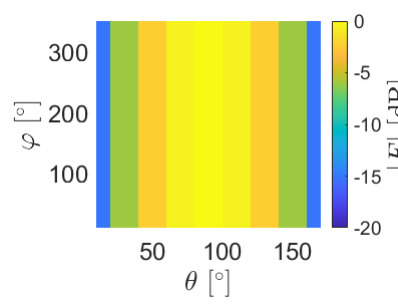

(a) FF, true

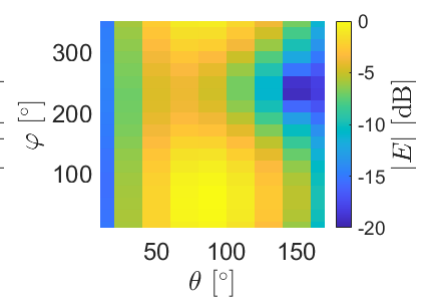

(b) FF, JCM

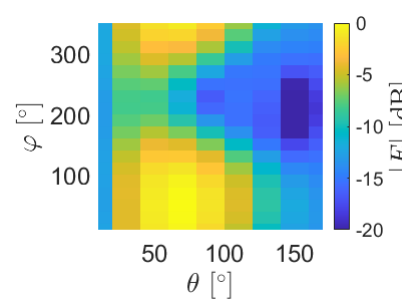

(c) FF, VPM

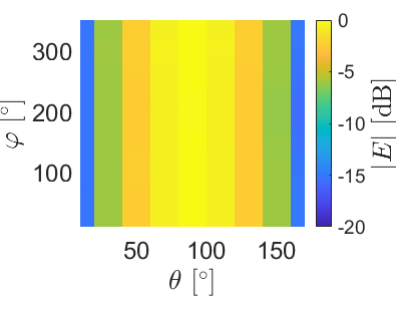

(d) FF, DSM
Fig. 6. Reconstructed far-field electric field magnitudes using the three main methods for the infinitesimal dipole problem with sectoral-view data.

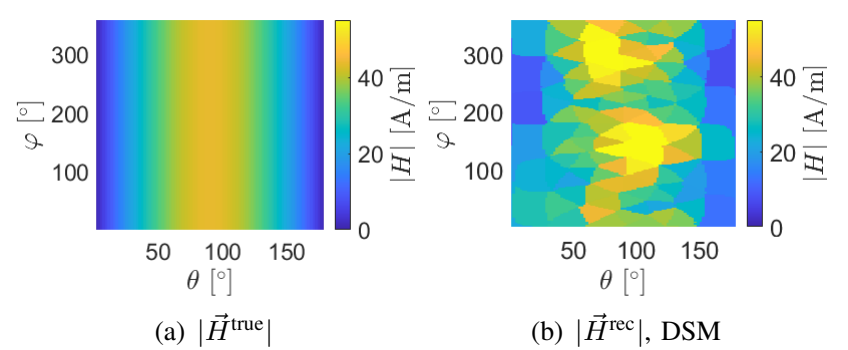

Fig. 7. True and reconstructed magnetic field magnitude on $\mathcal{R}^{-}$(DSM only) for the infinitesimal dipole problem with sectoral-view data.

radiating. Since the Krylov subspace regularization seeks a minimum-norm type of solution [14], these currents are not favored during the optimization process. Note also that in this case, changing the regularization weights does not improve the JCM and VPM; for example, if we relax the Krylov subspace regularization and allow a larger solution norm, the solution instead quickly destabilizes and is unable to reach the true solution. This also applies to the identity Tikhonov constraint, which unlike the first example does not obtain a relatively accurate far-field.

\section{Expanded Discussion of DSM Results}

Although the greater reconstruction accuracy of the DSM is expected, it can be disconcerting that the reconstructed solution is fully symmetric, whereas the data is notably non-symmetric. For this reason, we will hold an expanded discussion of the speculative advantages of the DSM. To begin, it is instructive to see the DSM's reconstructed currents, or fields, on $\mathcal{R}^{-}$; of these, the magnetic field magnitude has been plotted in Fig. 7. The immediate observation is the fact that the currents are distributed in almost an opposite fashion to the $\mathbf{y}$ currents for the JCM and VPM. For the JCM and VPM, the strongest fields are those nearest to the measured data; for the $\mathbf{x}$ currents of the DSM, it is the opposite. When propagated 
by $\mathbf{B}_{\mathrm{DSM}}$, the effect is to favor a similar distribution on $\mathbf{y}$, due to the relative proximity. Thus, from a purely causal point of view, it is the $\mathbf{x}$ currents which enforce the symmetry on the $\mathbf{y}$ currents.

We propose that by linking two otherwise independent vectors (i.e., each satisfies its own independent data equation), a strong restriction is imposed on the undesired non-radiating currents in $\mathbf{y}$. Alternatively, the exclusion or cancellation of desired non-radiating currents is not allowed by the unique formulation of the DSM. A result which supports this can be found in Fig. $5(\mathrm{~g})-(\mathrm{h})$, which shows the reconstruction results for the DSM-P; these are most similar to the JCM. As previously mentioned, the $\mathbf{y}$ currents in the DSM-P are decoupled from the data equation; thus, it is reasonable to speculate that it is the combination of two independent, but linked, data equations which provide the main advantage of the DSM. This is not unlike the behavior that can be seen in phaseless near-field measurements [4], [29]; in that case, two otherwise independent sets of measured field magnitudes are linked in order to recover the phase. The DSM strategy also appears to share similarities with the strategies used to prevent internal resonances in forward scattering problems, e.g., the combined field integral equation (CFIE) or the aptly named dual surface integral equation [30], [31]. Note that the issue of internal resonance is already addressed by any of the Love's constraints as implemented herein; thus, the physical interpretation of the DSM is different from these methods.

To reiterate, it appears that the linking of otherwise independent data provides additional information; thus, the unique formulation of the DSM can be thought as providing additional information which is not seen by the JCM and VPM. Since the ambiguity of inverse problems is caused by a lack of information, it is natural that the method which provides the most information will see the best performance. As a corollary, we see that the separation distance for the DSM should be larger than in the VPM, as this increases the independence between the two sets of currents. It may also be possible that the drastic performance of the DSM is due to the specific problem parameters, i.e., the difference being so large is partly a coincidence. For these reasons, a separate measurement example will be examined shortly. Nonetheless, we expect that the relative behavior of each method remains consistent with the previous results.

\section{E. Circular Horn Antenna}

In order to reinforce the results of the previous example, the three reconstruction methods will be applied to the case of a circular horn antenna operating at $8 \mathrm{GHz}$ and radiating along the $z$-axis. In certain practical cases, the reconstruction surface for the SRM is not closed; thus, half-spheres will be used for $\mathcal{R}, \mathcal{R}^{-}$, and $\mathcal{S}$ to see if this may affect the comparative results. See Fig. 8 for the (not-to-scale) visualization of this problem. The measured data on the half-sphere $\mathcal{S}$ of radius $r_{\mathcal{S}}=10 \mathrm{~cm} \sim 8 \lambda / 3$ was made by simulating the horn antenna in the ANSYS $^{\circledR}$ HFSS $^{\mathrm{TM}}$ software in ANSYS $^{\circledR}$ Electronics Desktop $^{\text {TM }} 2020 \mathrm{R} 1$. The main reconstruction surface $\mathcal{R}$ is a half-sphere of radius $r_{0}=4.5 \mathrm{~cm} \sim 6 \lambda / 5$. The maximum

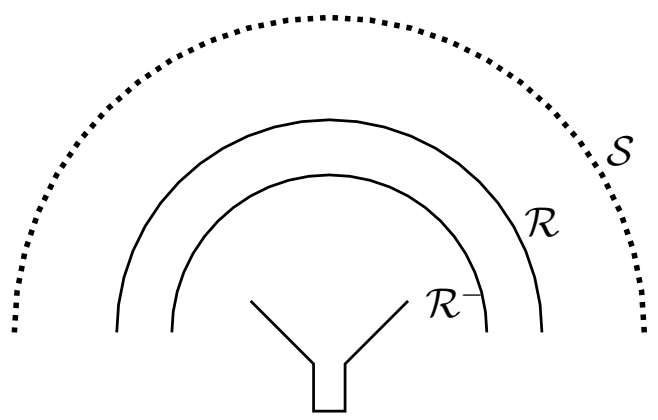

Fig. 8. Problem setup for the horn antenna example. The reconstruction and measurement surfaces are half-spheres, roughly centered on the horn antenna but biased towards the aperture. Figure not to scale.

mesh element size is again $l_{e}=\lambda / 10$. The auxiliary surface for the VPM is a sphere of radius $r_{\mathrm{VPM}}^{\prime}=r_{0}-l_{e}$, and the auxiliary surface for the DSM is a sphere of radius $r_{\mathrm{DSM}}^{\prime}=3.5 \mathrm{~cm} \sim r_{0}-\lambda / 4$. The testing functions for the Love's constraint have the same characteristics as in the previous example. The measured data has also been perturbed by varying levels of synthetic white noise. The noise level is defined as the maximum noise magnitude referred to the maximum field magnitude on $\mathcal{S}$.

In the previous example, the differences between the methods (i.e., their effect on the undesired non-radiating sources) was demonstrated by limiting the measured data to a sector. For this example, the case of under-sampled data will instead be investigated. As an estimate for the minimum sampling rate, we can look at the well-known formulas for conventional nearfield measurements [13]. Using the formulas for a spherical measurement, the angular sampling is approximately ${ }^{8} \Delta \theta=$ $\Delta \varphi=13^{\circ}$. The final sampling for the example was chosen as $\Delta \theta=20^{\circ}$ and $\Delta \varphi=60^{\circ}$, which results in a significantly under-sampled problem. Using this under-sampled measured data, the three main methods were again evaluated. The reconstructed field magnitudes for the methods, under $2 \%$ noise, can be found in Fig. 9 plotted over $\Delta \theta=\Delta \varphi=10^{\circ}$. Similarly to the case with the infinitesimal dipole, the DSM produces the most visually faithful results. In contrast, the JCM and VPM struggle to find an adequate solution; this is a testament to the proposition that these methods are more difficult to regularize. The combined magnitude and phase error defined in (49) is summarized in Table $\mathrm{V}$ for varying noise levels. Compared to the previous example, the DSM method has a much larger error; this is partly due to the increased approximation error from an open reconstruction surface. Nonetheless, the error values remain the lowest for the DSM and the field plots are visually faithful.

Next, we would like to focus on the reconstructed farfields, whose error is also found in Table V. Similarly to the second case for the infinitesimal dipole, the accuracy of the reconstructed currents is proportional to the far-field error. This is due to the presence of currents which are non-radiating with respect to the measured data, but otherwise arbitrary.

\footnotetext{
${ }^{8}$ This result is obtained by using the formula $\Delta \theta=\Delta \varphi=0.5 \lambda /(a+$ $\lambda$ ) [13], where $a$ is the radius of the minimum sphere which envelops the antenna ( $a$ is taken as $4.5 \mathrm{~cm}$ ).
} 


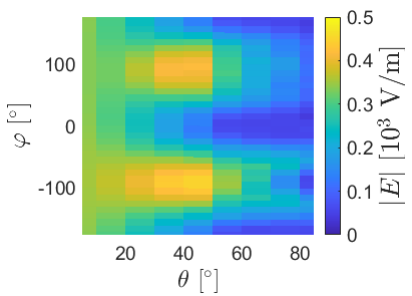

(a) $\left|\vec{E}^{\text {true }}\right|$

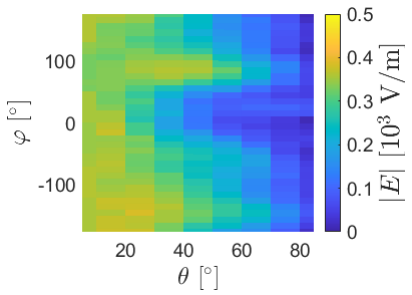

(c) $\left|\vec{E}^{\mathrm{rec}}\right|$, JCM

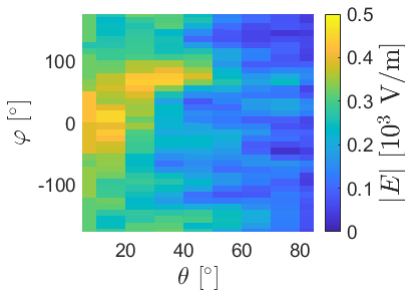

(e) $\left|\vec{E}^{\text {rec }}\right|$, VPM

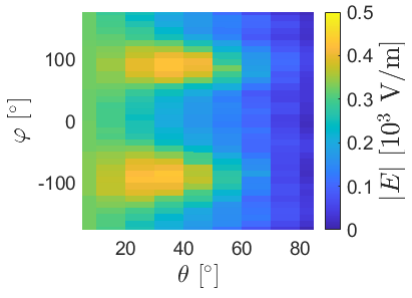

(g) $\left|\vec{E}^{\mathrm{rec}}\right|$, DSM

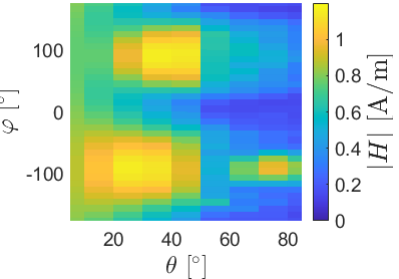

(b) $\left|\vec{H}^{\text {true }}\right|$

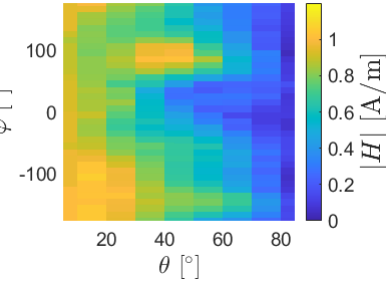

(d) $\left|\vec{H}^{\text {rec }}\right|$, JCM
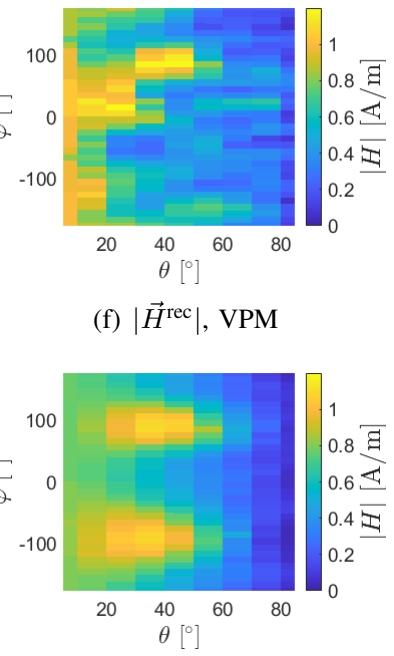

(h) $\left|\vec{H}^{\text {rec }}\right|$, DSM (f) $\left|\vec{H}^{\text {rec }}\right|$, VPM

Fig. 9. Field magnitudes on $\mathcal{R}$ for the horn antenna example.

TABLE V

RECONSTRUCTION ERRORS FOR THE HORN ANTENNA.

\begin{tabular}{|c|c|c|c|c|c|c|}
\hline & \multicolumn{6}{|c|}{ Noise Level } \\
\hline & \multicolumn{3}{|c|}{$2 \%$} & \multicolumn{3}{|c|}{$20 \%$} \\
\hline & $\epsilon_{E}$ & $\epsilon_{H}$ & $\epsilon_{\mathrm{FF}}$ & $\epsilon_{E}$ & $\epsilon_{H}$ & $\epsilon_{\mathrm{FF}}$ \\
\hline $\mathrm{JCM}$ & $39.1 \%$ & $46.3 \%$ & $17.8 \%$ & $44.7 \%$ & $53.1 \%$ & $21.2 \%$ \\
\hline VPM & $61.7 \%$ & $69.4 \%$ & $26.6 \%$ & $64.9 \%$ & $73.7 \%$ & $30.1 \%$ \\
\hline DSM & $29.3 \%$ & $33.9 \%$ & $10.6 \%$ & $34.6 \%$ & $41.5 \%$ & $12.2 \%$ \\
\hline
\end{tabular}

Since the measured data are under-sampled, these currents can produce an error in the far-field. To visualize these results, the normalized far-field under $2 \%$ noise has been plotted over two perpendicular cuts along the $x-z$ and $y-z$ planes in Fig. 10. Along the $x-z$ cut, each method is fairly similar; along the $y-z$ cut, we can see the improvement that the DSM provides in this example. (Note that the side lobe on the $y$ - $z$ cut is caused by the coaxial feed to the horn antenna.) In addition, the reconstructed main beam direction is shifted for both the VPM and JCM, whereas there is no shift for the DSM.

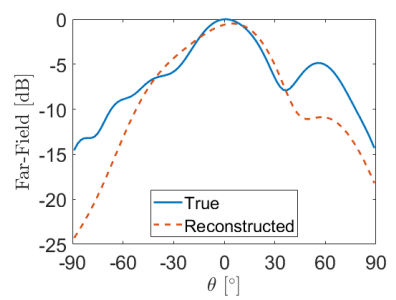

(a) FF $y$ - $z$ cut, JCM

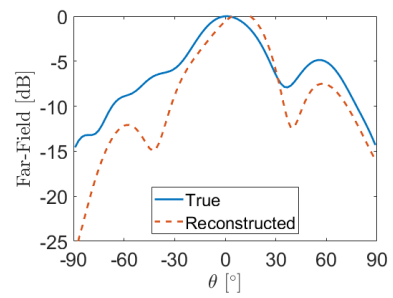

(c) FF $y$ - $z$ cut, VPM

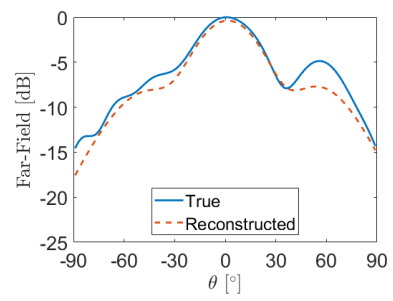

(e) FF $y-z$ cut, DSM

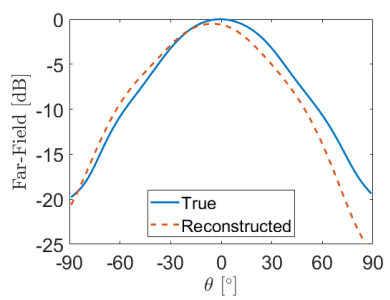

(b) FF $x-z$ cut, JCM

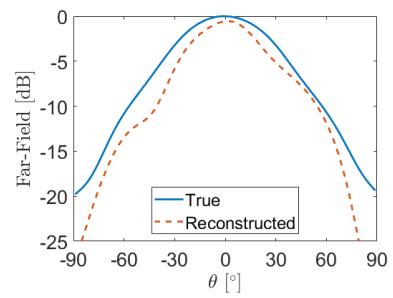

(d) FF $x-z$ cut, VPM

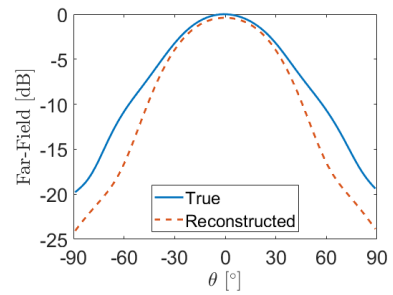

(f) FF $x-z$ cut, DSM
Fig. 10. Far-field (FF) cuts for the horn antenna example. For the $y$ - $z$ cut, a positive $\theta$ value corresponds to $\phi=90^{\circ}$ and a negative $\theta$ value is the equivalent positive $\theta$ corresponding to $\phi=-90^{\circ}$. For the $x-z$ cut, a positive $\theta$ value corresponds to $\phi=180^{\circ}$ and a negative $\theta$ value is the equivalent positive $\theta$ corresponding to $\phi=0^{\circ}$.

\section{DISCUSSION}

The purpose of the examples presented herein is to demonstrate the usefulness of the Love's constraint and the importance of a restrictive condition. In the initial example using the infinitesimal dipole, the Love's methods produced markedly better diagnostic results than the identity Tikhonov regularizer; in this case, the restriction on the undesired non-radiating currents makes it easy to tune the subsequent regularization and obtain a reasonable solution. Among the Love's methods, there were differences, but each method produced reasonable results. In the second example using the infinitesimal dipole, the increased ambiguity caused by the lack of measured data resulted in additional non-radiating currents. Only the DSM, with its more restrictive constraint, was able to obtain a reasonable solution. In addition, we were able to see that the additional data equation in the DSM is also important for restricting the problem, as the DSM-P was unable to obtain a reasonable solution. Furthermore, this example also demonstrated that there exist non-radiating currents, defined with respect to $\mathcal{S}$, which can affect the far-field results. Thus, the Love's constraint can also be useful for obtaining an accurate far-field. Finally, the third example using the circular horn antenna was a more practical scenario to reinforce the findings in the second example. Once again, the DSM was better able to obtain a reasonable solution in terms of the 
reconstructed fields on $\mathcal{R}$ and the far-field.

It is important to reinforce that the examples provided were carefully chosen to magnify the differences that can occur between all of the methods. When the measured data were well sampled over a closed surface in the first example, these differences were present but minimal. In this case, the Love's condition is primarily useful only for antenna diagnostics. Furthermore, for antenna characterization, the identity Tikhonov constraint, or any other general regularization, will provide results which are as good or better than the Love's methods, since it is the data misfit cost functional which regulates the far-field accuracy by the uniqueness theorem. However, if this condition is not met, the Love's constraint, or in particular the DSM, can be useful for antenna characterization since the true Love's currents are guaranteed to produce the correct far-field. It remains to be seen if there are practical scenarios at which point the use of the DSM is encouraged.

\section{CONCLUSION}

The source reconstruction method is a powerful tool for the purposes of antenna characterization and diagnostics. Since this technique represents an inverse problem, it is susceptible to the complications of inverse problems; in this context, there are many possible solutions, represented by non-radiating currents, which adequately satisfy the near-field measured data. This effect is an issue for antenna diagnostics and can be an issue for antenna characterization, as was demonstrated herein. In order to mitigate these issues, regularization techniques are employed which ideally remove the undesired non-radiating currents. However, a general regularizer, such as an identity Tikhonov constraint, cannot typically differentiate between desired and undesired non-radiating currents. Thus, there is a trade-off between solution accuracy and stability, which is adjusted by a regularization weight. Alternatively, a Love's constraint may be employed, which is known to be satisfied by the true solution. The effect of this constraint is a removal of a portion of undesired non-radiating currents. Although it does not completely remove all of these currents, any subsequent regularization is made easier, which can result in a more accurate solution. By the equivalence principle, there are at least three main methods for formulating the Love's constraint. Under ideal conditions, each method is equivalent; under non-ideal conditions, the methods can differ. Furthermore, each method provides its own advantages and disadvantages. Although the VPM provides a less restrictive condition, it is conceptually the easiest of the three methods to implement. The DSM provides a more restrictive condition, but to obtain the full benefits of the method we require an additional vector of unknowns and an additional data equation. Lastly, the JCM provides a middle-of-the-road restriction, but is very efficient under the projected form.

We would like to conclude by emphasizing that the differences between the Love's methods manifest themselves most strongly in non-ideal problems. In many real-life scenarios, any of the methods presented should produce adequate results. However, if a non-ideal scenario occurs, an understanding of the advantages and disadvantages of the Love's methods will be useful for the prospective antenna engineer.

\section{APPENDIX A \\ VECTOR FIELD REPRESENTATION}

The vector field representation provides an intuitive yet mathematically consistent way to analyze the various forms of the Love's condition constraint. This representation is obtained by applying the testing procedure of the MoM to the analytical equations in Section IV. To begin, we will assume that we are dealing with the JCM and that the testing functions in the MoM scheme are tangential vectors on $\mathcal{R}$ in the point form. Since the tangential vector (at a given point) can be expressed as the sum of two orthogonal vectors in the same tangential plane, the testing functions $\left\{\vec{T}\left(\vec{r}_{p, q}\right)\right\}$ can be expressed as

$$
\begin{aligned}
& \left\{\vec{T}\left(\vec{r}_{p, q}\right)\right\}_{i, 1}=\hat{t}_{i, 1} \delta\left(\vec{r}-\vec{r}_{i, 1}\right), \\
& \left\{\vec{T}\left(\vec{r}_{p, q}\right)\right\}_{i, 2}=\hat{t}_{i, 2} \delta\left(\vec{r}-\vec{r}_{i, 2}\right),
\end{aligned}
$$

where $\hat{t}$ is the orthogonal vector, $i$ is an index for the distinct testing locations, and the subscript 1 or 2 denotes one of the orthogonal components. Note that $\vec{r}_{i, 1}=\vec{r}_{i, 2}=\vec{r}_{i}$. If we further assume that $\hat{t}_{i, 1} \times \hat{t}_{i, 2}=\hat{n}$, the boundary conditions on $\mathcal{R}$ in (1) can be expanded as

$$
\begin{gathered}
-\hat{t}_{i, 1} \cdot \vec{J}\left(\vec{r}_{i}\right)=\hat{t}_{i, 2} \cdot \vec{H}_{2}\left(\vec{r}_{i}\right)-\hat{t}_{i, 2} \cdot \vec{H}_{1}\left(\vec{r}_{i}\right), \\
\hat{t}_{i, 2} \cdot \vec{J}\left(\vec{r}_{i}\right)=\hat{t}_{i, 1} \cdot \vec{H}_{2}\left(\vec{r}_{i}\right)-\hat{t}_{i, 1} \cdot \vec{H}_{1}\left(\vec{r}_{i}\right), \\
\hat{t}_{i, 1} \cdot \vec{M}\left(\vec{r}_{i}\right)=\hat{t}_{i, 2} \cdot \vec{E}_{2}\left(\vec{r}_{i}\right)-\hat{t}_{i, 2} \cdot \vec{E}_{1}\left(\vec{r}_{i}\right), \\
-\hat{t}_{i, 2} \cdot \vec{M}\left(\vec{r}_{i}\right)=\hat{t}_{i, 1} \cdot \vec{E}_{2}\left(\vec{r}_{i}\right)-\hat{t}_{i, 1} \cdot \vec{E}_{1}\left(\vec{r}_{i}\right),
\end{gathered}
$$

where we have simply performed an inner product with the testing functions. These relations can be generalized as

$$
\gamma_{i}^{j}=\psi_{2, i}^{j}-\psi_{1, i}^{j},
$$

where

$$
\begin{gathered}
\gamma_{i}^{j}= \begin{cases}\hat{t}_{i, 1} \cdot \vec{J}\left(\vec{r}_{i}\right), & j=1 \\
\hat{t}_{i, 2} \cdot \vec{J}\left(\vec{r}_{i}\right), & j=2 \\
\hat{t}_{i, 1} \cdot \vec{M}\left(\vec{r}_{i}\right), & j=3 \\
\hat{t}_{i, 2} \cdot \vec{M}\left(\vec{r}_{i}\right), & j=4\end{cases} \\
\psi_{m, i}^{j}=\left\{\begin{array}{rll}
-\hat{t}_{i, 2} \cdot \vec{H}_{m}\left(\vec{r}_{i}\right), & j=1, & m \in\{1,2\} \\
\hat{t}_{i, 1} \cdot \vec{H}_{m}\left(\vec{r}_{i}\right), & j=2, & m \in\{1,2\} \\
\hat{t}_{i, 2} \cdot \vec{E}_{m}\left(\vec{r}_{i}\right), & j=3, & m \in\{1,2\} \\
-\hat{t}_{i, 1} \cdot \vec{E}_{m}\left(\vec{r}_{i}\right), & j=4, & m \in\{1,2\}
\end{array}\right.
\end{gathered}
$$

If the testing procedure is applied to (16) and (17), $\gamma_{i}^{j} / 2$ terms will appear on the left-hand side, which is equivalent to $\tilde{\mathbf{I}} \mathbf{y} / 2$ by (18). Therefore, the product $\tilde{\mathbf{I}} \mathbf{y}$ is just the vector of all possible $\gamma_{i}^{j}$; by (57), the product may be written as

$$
\tilde{\mathbf{I}} \mathbf{y} \rightarrow \boldsymbol{\psi}_{2}-\boldsymbol{\psi}_{1},
$$

where the bold $\psi_{2}$ and $\psi_{1}$ are vectors containing their corresponding scalar quantities. Following this, we would next like to write a similar expression for the product $\mathbf{B}_{\text {JСM }} \mathbf{y}$. We first recognize that the output of the operator is the averaged tangential field, where the magnetic field is positive, and the electric field is negative (see (14)-(18)). Applying the point 
testing functions to the analytical output of $\mathbf{B}_{\mathrm{JCM}} \mathbf{y}$ results in inner product terms $P_{i}^{j}$ of the form

$$
\begin{gathered}
P_{i}^{1}=\hat{t}_{i, 1} \cdot\left\{\hat{n} \times \vec{f}_{H_{i}}\right\} / 2, \\
P_{i}^{2}=\hat{t}_{i, 2} \cdot\left\{\hat{n} \times \vec{f}_{H_{i}}\right\} / 2, \\
P_{i}^{3}=-\hat{t}_{i, 1} \cdot\left\{\hat{n} \times \vec{f}_{E_{i}}\right\} / 2, \\
P_{i}^{4}=-\hat{t}_{i, 2} \cdot\left\{\hat{n} \times \vec{f}_{E_{i}}\right\} / 2,
\end{gathered}
$$

where

$$
\begin{gathered}
\left.\vec{f}_{H_{i}}=\left(\left[\left(\vec{H}_{2}\left(\vec{r}_{i}\right) \cdot \hat{t}_{i, 1}\right) \hat{t}_{i, 1}\right)+\left(\vec{H}_{1}\left(\vec{r}_{i}\right) \cdot \hat{t}_{i, 1}\right) \hat{t}_{i, 1}\right)\right] \\
\left.\left.\left.\quad+\left[\left(\vec{H}_{2}\left(\vec{r}_{i}\right) \cdot \hat{t}_{i, 2}\right) \hat{t}_{i, 2}\right)+\left(\vec{H}_{1}\left(\vec{r}_{i}\right) \cdot \hat{t}_{i, 2}\right) \hat{t}_{i, 2}\right)\right]\right), \\
\left.\vec{f}_{E_{i}}=\left(\left[\left(\vec{E}_{2}\left(\vec{r}_{i}\right) \cdot \hat{t}_{i, 1}\right) \hat{t}_{i, 1}\right)+\left(\vec{E}_{1}\left(\vec{r}_{i}\right) \cdot \hat{t}_{i, 1}\right) \hat{t}_{i, 1}\right)\right] \\
\left.\left.\left.\quad+\left[\left(\vec{E}_{2}\left(\vec{r}_{i}\right) \cdot \hat{t}_{i, 2}\right) \hat{t}_{i, 2}\right)+\left(\vec{E}_{1}\left(\vec{r}_{i}\right) \cdot \hat{t}_{i, 2}\right) \hat{t}_{i, 2}\right)\right]\right),
\end{gathered}
$$

which is just the decomposition of the field into orthogonal components. (Note that the normal component of the field is removed by the $\hat{n} \times($.$) operation.) Employing \hat{t}_{i, 1} \times \hat{t}_{i, 2}=\hat{n}$, the inner products can be further simplified into

$$
\begin{gathered}
P_{i}^{1}=-\left\{\hat{t}_{i, 2} \cdot \vec{H}_{2}\left(\vec{r}_{i}\right)+\hat{t}_{i, 2} \cdot \vec{H}_{1}\left(\vec{r}_{i}\right)\right\} / 2, \\
P_{i}^{2}=\left\{\hat{t}_{i, 1} \cdot \vec{H}_{2}\left(\vec{r}_{i}\right)+\hat{t}_{i, 1} \cdot \vec{H}_{1}\left(\vec{r}_{i}\right)\right\} / 2, \\
P_{i}^{3}=\left\{\hat{t}_{i, 2} \cdot \vec{E}_{2}\left(\vec{r}_{i}\right)+\hat{t}_{i, 2} \cdot \vec{E}_{1}\left(\vec{r}_{i}\right)\right\} / 2, \\
P_{i}^{4}=-\left\{\hat{t}_{i, 1} \cdot \vec{E}_{2}\left(\vec{r}_{i}\right)+\hat{t}_{i, 1} \cdot \vec{E}_{1}\left(\vec{r}_{i}\right)\right\} / 2 .
\end{gathered}
$$

Using the definitions in (59), the above can be represented as

$$
P_{i}^{j}=\left\{\psi_{2, i}^{j}+\psi_{1, i}^{j}\right\} / 2
$$

or in other words

$$
\mathbf{B}_{\mathrm{JCM}} \mathbf{y} \rightarrow \frac{\boldsymbol{\psi}_{2}+\boldsymbol{\psi}_{1}}{2}
$$

The usefulness of this representation is that we can quickly and intuitively evaluate the side constraint for each of the methods presented in this paper.

For more general testing functions, we can consider the linear dependence of the side constraint under different sets of testing functions. First assume that we have the side constraint matrix $\mathbf{B}_{\mathrm{JCM}, 1}$, which is constructed with point testing functions as shown and $\mathbf{B}_{\mathrm{JCM}, 1} \in \mathbb{C}^{m_{1} \times N}$ with $m_{1} \geq N$. Using a different set of testing functions, we construct the matrix $\mathbf{B}_{\mathrm{JCM}, 2}$ where $\mathbf{B}_{\mathrm{JCM}, 2} \in \mathbb{C}^{m_{2} \times N}$ with $m_{2} \geq N$. If we assume that each matrix contains exactly $N$ linearly independent rows, then each matrix forms a basis for $\mathbb{C}^{N}$. Thus, it is possible to employ an elementary matrix $\mathbf{L}_{12} \in \mathbb{C}^{m_{2} \times m_{1}}$ to transform $\mathbf{B}_{\mathrm{JCM}, 1}$ into $\mathbf{B}_{\mathrm{JCM}, 2}$, i.e.

$$
\mathbf{L}_{12} \mathbf{B}_{\mathrm{JCM}, 1}=\mathbf{B}_{\mathrm{JCM}, 2} .
$$

Having already established that $\mathbf{B}_{\mathrm{JCM}, 1} \mathbf{y}=\left(\boldsymbol{\psi}_{2}+\boldsymbol{\psi}_{1}\right) / 2$, it follows that the product $\mathbf{B}_{\mathrm{JCM}, 2} \mathbf{y}$ is

$$
\mathbf{B}_{\mathrm{JCM}, 2} \mathbf{y}=\mathbf{L}_{12}\left(\frac{\boldsymbol{\psi}_{2}+\boldsymbol{\psi}_{1}}{2}\right) .
$$

The same transformation can be made for the product $\tilde{\mathbf{I}}_{2} \mathbf{y}$ by employing the same elementary matrix. Thus, the JCM constraint under different testing functions may be written as

$$
\begin{gathered}
\mathbf{0}=\mathbf{L}_{12}\left(\mathbf{B}_{\mathrm{JCM}, 1} \mathbf{y}-\frac{\tilde{\mathbf{I}}_{1} \mathbf{y}}{2}\right) \\
\mathbf{0}=\mathbf{L}_{12}\left(\frac{\boldsymbol{\psi}_{2}+\boldsymbol{\psi}_{1}}{2}-\frac{\boldsymbol{\psi}_{2}-\boldsymbol{\psi}_{1}}{2}\right) \Longrightarrow \mathbf{0}=\mathbf{L}_{12}\left(\boldsymbol{\psi}_{1}\right) .
\end{gathered}
$$

The end result is that the required cancellations in Section $\mathrm{V}$ will occur, but the remainder will be weighted by $\mathbf{L}_{12}$. Numerically, the two representations may have small behavioral differences, but conceptually they are the same (i.e., they contain the same information). Thus, we consider the previous results to be an appropriate (conceptual) representation of all general side constraint matrices which are of sufficient rank.

\section{REFERENCES}

[1] A. Yaghjian, "An overview of near-field antenna measurements," IEEE Trans. Antennas Propag., vol. 34, no. 1, pp. 30-45, 1986.

[2] C. Parini, S. Gregson, and D. J. van Rensburg, Theory and Practice of Modern Antenna Range Measurements. IET, 2014.

[3] T. F. Eibert and C. H. Schmidt, "Multilevel fast multipole accelerated inverse equivalent current method employing RaoWiltonGlisson discretization of electric and magnetic surface currents," IEEE Transactions on Antennas and Propagation, vol. 57, no. 4, pp. 1178-1185, 2009.

[4] T. Brown, I. Jeffrey, and P. Mojabi, "Multiplicatively regularized source reconstruction method for phaseless planar near-field antenna measurements," IEEE Trans. Antennas Propag, vol. 65, no. 4, pp. 2020-2031, April 2017.

[5] A. Taaghol and T. K. Sarkar, "Near-field to near/far-field transformation for arbitrary near-field geometry, utilizing an equivalent magnetic current," IEEE Transactions on Electromagnetic Compatibility, vol. 38, no. 3, pp. 536-542, Aug 1996.

[6] Y. Alvarez Lopez, F. Las-Heras Andres, M. R. Pino, and T. K. Sarkar, "An improved super-resolution source reconstruction method," IEEE Trans. Instrum. Meas., vol. 58, no. 11, pp. 3855-3866, 2009.

[7] J. L. A. Quijano and G. Vecchi, "Improved-accuracy source reconstruction on arbitrary 3-D surfaces," IEEE Antennas and Wireless Propagation Letters, vol. 8, pp. 1046-1049, 2009.

[8] A. J. Devaney and G. C. Sherman, "Nonuniqueness in inverse source and scattering problems," IEEE Trans. Antennas Propag., vol. 30, no. 5, pp. 1034-1042, Sep 1982.

[9] J. L. A. Quijano and G. Vecchi, "Field and source equivalence in source reconstruction on 3D surfaces," Progress In Electromagnetics Research, vol. 103, pp. 67-100, 2010.

[10] J. Kornprobst, R. A. M. Mauermayer, E. Kl, and T. F. Eibert, "An inverse equivalent surface current solver with zero-field enforcement by lefthand side Caldern projection," in 2019 13th European Conference on Antennas and Propagation (EuCAP), 2019, pp. 1-3.

[11] J. Kornprobst, J. Knapp, R. A. M. Mauermayer, O. Neitz, A. Paulus, and T. F. Eibert, "Accuracy and conditioning of surface-source based near-field to far-field transformations," IEEE Transactions on Antennas and Propagation (Early Access), 2021.

[12] R. F. Harrington, Time-Harmonic Electromagnetic Fields. IEEE-Press, 2001. [Online]. Available: http://dx.doi.org/10.1109/9780470546710

[13] C. A. Balanis, Antenna theory: analysis and design. Wiley-Interscience, 2005.

[14] P. C. Hansen, "Numerical tools for analysis and solution of Fredholm integral equations of the first kind," Inverse Probl., vol. 8, pp. 849-872, 1992.

[15] T. S. Shores, Applied Linear Algebra and Matrix Analysis, 2nd ed., ser. Undergraduate Texts in Mathematics. Cham: Springer International Publishing.

[16] R. C. Aster, Parameter estimation and inverse problems, ser. International geophysics series; v. 90. Amsterdam: Elsevier Academic Press.

[17] A. Grbic, L. Jiang, and R. Merlin, "Near-field plates: Subdiffraction focusing with patterned surfaces," Science, vol. 320, no. 5875, pp. 511513,2008 
[18] M. Ili, I. W. Turner, and Y. Saad, "Linear system solution by null-space approximation and projection (SNAP)," Numerical Linear Algebra with Applications, vol. 14, no. 1, pp. 61-82, 2007. [Online]. Available: https://onlinelibrary.wiley.com/doi/abs/10.1002/nla.509

[19] T. K. Jensen, "Stabilization algorithms for large-scale problems," Ph.D. dissertation, Technical University of Denmark, Kongens Lyngby, Denmark, 2006

[20] P. Mojabi and J. LoVetri, "Overview and classification of some regularization techniques for the Gauss-Newton inversion method applied to inverse scattering problems," IEEE Transactions on Antennas and Propagation, vol. 57, no. 9, pp. 2658-2665, 2009.

[21] R. L. Parker, Geophysical inverse theory. Princeton, N.J: Princeton University Press, 1994.

[22] T. Brown, C. Narendra, Y. Vahabzadeh, C. Caloz, and P. Mojabi, "On the use of electromagnetic inversion for metasurface design," IEEE Trans. Antennas Propag., vol. 68, no. 3, pp. 1812-1824, 2020.

[23] M. Phaneuf and P. Mojabi, "Electromagnetic inversion for noninvasive specific absorption rate characterization," IEEE J. Electromagn., RF, Microw. Med. Biol. (Early Access), 2020.

[24] J. L. Volakis, Integral Equation Methods for Electromagnetics. Raleigh, NC: SciTech Pub., 2012.

[25] P. C. Hansen, M. E. Kilmer, and R. H. Kjeldsen, "Exploiting residual information in the parameter choice for discrete ill-posed problems," BIT Numerical Mathematics, vol. 46, pp. 41-59, 2006.

[26] L. Li, K. Wang, and T. F. Eibert, "A projection height independent adaptive radial-angular- $r^{2}$ transformation for singular integrals," IEEE Trans. Antennas Propag., vol. 62, no. 10, pp. 5381-5386, Oct 2014.

[27] F. Vipiana, A. Polemi, S. Maci, and G. Vecchi, "A mesh-adapted closed-form regular kernel for 3D singular integral equations," IEEE Transactions on Antennas and Propagation, vol. 56, no. 6, pp. 16871698, 2008.

[28] W. C. Gibson, The method of moments in electromagnetics, 2nd ed. Boca Raton, FL: CRC Press/Taylor \& Francis Group.

[29] S. F. Razavi and Y. Rahmat-Samii, "Resilience to probe-positioning errors in planar phaseless near-field measurements," IEEE Transactions on Antennas and Propagation, vol. 58, no. 8, pp. 2632-2640, 2010.

[30] W. Chew, M. S. Tong, and B. hu, Integral Equation Methods for Electromagnetic and Elastic Waves, 2008.

[31] R. A. Shore and A. D. Yaghjian, "Dual-surface integral equations in electromagnetic scattering," IEEE Transactions on Antennas and Propagation, vol. 53, no. 5, pp. 1706-1709, 2005. 